\title{
Experimental Investigation and Quantitative Calculation of the Degree of Hydration and Products in Fly Ash-Cement Mixtures
}

\author{
Zhiyong Liu, ${ }^{1,2}$ Dong Xu, ${ }^{1}$ and Yunsheng Zhang ${ }^{3}$ \\ ${ }^{1}$ State Key Laboratory of Geomechanics \& Deep Underground Engineering, China University of Mining and Technology, \\ Xuzhou 221116, China \\ ${ }^{2}$ Jiangsu Key Laboratory of Environmental Impact and Structural Safety in Engineering, China University of Mining and Technology, \\ Xuzhou 221116, China \\ ${ }^{3}$ Jiangsu Key Laboratory for Construction Materials, Southeast University, Nanjing 211189, China
}

Correspondence should be addressed to Zhiyong Liu; liuzhiyong0728@163.com

Received 10 July 2016; Revised 7 October 2016; Accepted 30 November 2016; Published 9 January 2017

Academic Editor: Jun Liu

Copyright (C) 2017 Zhiyong Liu et al. This is an open access article distributed under the Creative Commons Attribution License, which permits unrestricted use, distribution, and reproduction in any medium, provided the original work is properly cited.

\begin{abstract}
To explore the hydration process of fly ash-cement blended mixtures, the degrees of the fly ash and cement reactions as well as the content of nonevaporated water were determined at various water to binder ratios, curing ages, and fly ash incorporation amounts. An equation describing the relationship between the degree of hydration and the effective water to binder ratio was established based on the experimental results. In addition, a simplified scheme describing a model of the degree of reaction in fly ash-cement mixtures is proposed. Finally, using reaction stoichiometry, quantitative equations for the hydration products of fly ash-cement blended pastes are proposed by considering the hydration reactions of fly ash and cement as well as their interactions. The predicted results of the enhanced degree of cement hydration, content of calcium hydroxide $(\mathrm{CH})$, and porosity are consistent with the experimental data.
\end{abstract}

\section{Introduction}

Along with the scale of industrial production, the content requirements of concrete used in construction are increasing; at the same time, due to the shortage of energy and mineral resources, the demand for high-performance cement has become much greater. One important way of addressing this demand is to produce high-performance cement, regulated supplementary cementitious materials, and high cementitious clinker composites [1-3]. Recently, supplementary cementitious materials have been widely used in concrete, either in blended cements or added separately in cementbased materials [4]. These supplementary cementitious materials, which are powdery industrial by-products, such as fly ash from coal combustion; silica fume from ferrosilicon alloy manufacture; and blast-furnace slag, a byproduct of pig iron production, have latent hydraulic activity or volcanic properties. Fly ash is known as a pozzolana that possesses little or no cementitious value [5], and it accelerates the rate of cement hydration by a surface absorption effect [6] and a heterogeneous nucleation effect [7]. Therefore, fly ash can significantly improve the cured mechanical properties [8] and durability $[9,10]$ of cement-based materials. In addition, due to their spherical geometry, fly ash particles can improve the workability of fresh pastes [11]. Accordingly, fly ash, mixed with cement-based composite materials, greatly improves the performance of concrete and makes the microstructure of the composite material more complex.

To accurately investigate the volume fraction of each phase in cement-fly ash mixtures, the degree of hydration of cement and fly ash must first be determined. The experimental methods for measuring the degree of hydration of pure cement mainly include the hydration heat method [1214], the $\mathrm{CH}$ measurement method $[15,16]$, the nonevaporated water method $[5,17]$, the backscattered electron microscopy method $[18,19]$, and the XRD quantitative analysis method [20-22]. For the fly ash-cement composite cementitious system, because of the simultaneous presence of the cement hydration reaction and the fly ash reaction, the amount of nonevaporated water and $\mathrm{CH}$ water hydration products 
TABLE 1: The chemical composition of raw materials, wt.\%.

\begin{tabular}{lcccccccccccc}
\hline Material & $\mathrm{SiO}_{2}$ & $\mathrm{TiO}_{2}$ & $\mathrm{Al}_{2} \mathrm{O}_{3}$ & $\mathrm{Fe}_{2} \mathrm{O}_{3}$ & $\mathrm{MnO}$ & $\mathrm{MgO}$ & $\mathrm{CaO}$ & $\mathrm{Na}_{2} \mathrm{O}$ & $\mathrm{K}_{2} \mathrm{O}$ & $\mathrm{SO}_{3}$ & $\mathrm{~L} . \mathrm{O} . \mathrm{I}$ & $\sum$ \\
\hline Cement & 21.68 & 0.28 & 5.64 & 4.22 & 0.12 & 0.81 & 64.89 & 0.2 & 0.76 & 2.51 & 0.54 & 99.14 \\
Fly ash & 47.86 & 1.25 & 32.5 & 4.52 & 0.06 & 1.05 & 4.09 & 0.55 & 1.62 & 0.00 & 6.34 & 100.04 \\
\hline
\end{tabular}

cannot be used to distinguish the hydration degrees of the individual components. To accurately study the hydration degree of cement and the reaction degree of fly ash in fly ash-cement mixtures, Suprenant and Papadopoulos [23] tested the degree of reaction of fly ash-cement blended pastes using selective dissolution by hydrochloric acid. Lam et al. [5] studied the hydration process of fly ash-cement composites and measured the hydration degree of cement and the reaction degree of fly ash in composite materials by determining the chemically combined water and using selective dissolution by hydrochloric acid. Bentz et al. [24] studied the fly ash reaction in two different blended cementfly ash systems using a selective dissolution technique based on ethylene diamine tetra acetic acid (EDTA) combined with $\mathrm{NaOH}$, diluted $\mathrm{NaOH}$ solution, and portlandite content. For hydration models of fly ash blended cement, a kinetic hydration model [25] and a synthetic model [26] were used to simulate hydration of cement-fly ash blends based on a multicomponent concept, respectively.

However, the microstructures of fly ash-cement mixtures are complex and have not been characterized quantitatively. The unhydrated particles, the main hydration product, and calcium silicate hydrate (C-S-H) are complex and change with time, temperature, water to cement ratio, and fly ash incorporation. This paper proposes a hydration model for fly ash-cement blended pastes that is based on the reaction degree and hydration products. This model is not only related to the hydration reactions of fly ash and cement but is also influenced by their interaction.

\section{Experimental Procedures}

2.1. Materials. The mass ratio of Portland cement clinker and gypsum was set at $95: 5$. These components were mixed and milled to a specific surface area of $310 \mathrm{~m}^{2} / \mathrm{kg}$. Fly ash, similar to ASTM Class F fly ash, was supplied by the Nanjing Thermal Power Plant. The cement and fly ash were dried at $105^{\circ} \mathrm{C}$ and then sieved to remove the coarse particles larger than $0.08 \mathrm{~mm}$. Their chemical compositions are shown in Table 1.

\subsection{Methods}

2.2.1. Sample Preparation. The mix proportions of blended materials are listed in Table 2. The fresh cement paste was put into sealed bags and held to a predetermined age in a standard curing chamber $\left(20^{\circ} \mathrm{C}\right.$ and $95 \%$ humidity). To prevent bleeding and layering of the pastes, the sealed bags were inverted every 15 minutes prior to the initial setting of the fly ash-cement mixtures.
TABLE 2: Mix proportions of blended materials, wt.\%

\begin{tabular}{lccc}
\hline Sample number & Cement & Fly ash & W/B \\
\hline PC & 100 & 0 & $0.3,0.4,0.5$ \\
FA 10 & 90 & 10 & 0.5 \\
FA 30 & 70 & 30 & 0.5 \\
FA 40 & 60 & 40 & 0.5 \\
FA 50 & 50 & 50 & $0.3,0.35,0.4,0.45$, \\
& & & 0.5 \\
\hline
\end{tabular}

Note: $\mathrm{W} / \mathrm{B}$ is water to binder ratio.

2.2.2. Determination of the Quantity of Nonevaporated Water. The hardened pastes were crushed and mixed with ethanol, and then approximately $1 \mathrm{~g}$ of sample was weighed (accurate to $0.1 \mathrm{mg}$ ) after drying. The initial moisture contents of the samples were recorded as $m_{0}$, and the samples were then heated to $900^{\circ} \mathrm{C}$ at $10^{\circ} \mathrm{C} / \mathrm{min}$ in a high-temperature furnace and held at this temperature for 30 minutes. Next, the pastes were taken out, cooled in the dryer, and weighed using an electronic balance. Finally, the masses of the samples were recorded as $m_{900{ }^{\circ} \mathrm{C}}$. Each group of samples was analyzed 3 times in parallel tests, and the average value was determined. The nonevaporated water values of the hardened pastes were calculated by the following equation:

$$
W_{n}=\frac{L-L_{\mathrm{C}}}{1-L_{\mathrm{C}}}
$$

where $W_{n}$ is the quantity of nonevaporated water, $L$ is loss on ignition, and $L=\left(m_{0}-m_{900^{\circ} \mathrm{C}}\right) / m_{0} . L_{\mathrm{C}}$ is loss on ignition of raw materials.

\subsubsection{Measurement of Degree of Reaction}

(1) Degree of the Cement Reaction. Based on the Bogue composition of the cement calculated using the oxide contents and the reported chemically bound water contents of the compounds [27], the degree of cement hydration in a pure cement paste can be calculated using the following equation:

$$
\alpha_{\mathrm{C}}=\frac{100 \times W_{n}}{0.23}
$$

where $\alpha_{\mathrm{C}}$ is the hydration degree of cement, \%.

(2) Degree of the Fly Ash Reaction. The samples of hardened paste were crushed and soaked for $25 \mathrm{~min}$ in isopropyl alcohol. The samples were milled so that all passed through a $0.08 \mathrm{~mm}$ sieve that was equipped with ethanol and a vacuum filter. After filtration, the powder samples were dried for $24 \mathrm{~h}$ 
at 80 to $200 \mathrm{kPa}$ and $105^{\circ} \mathrm{C}$ in a soda lime vacuum oven. The determination of the degree of reaction of the fly ash was based on a selective dissolution procedure using a picric acidmethanol solution and water [28].

\section{Equations for Quantitative Calculations}

3.1. Reaction Degree Model of Fly Ash-Cement Mixtures. Powers [29] considered that the nonevaporated water of pure cement paste is an important indicator of the degree of the cement reaction. The nonevaporated water ratio of hydration at different ages $\left(W_{n,(t)}\right)$ and the cement fully hydrated value $\left(W_{n,(\infty)}=0.23\right)$ characterized the degree of the cement reaction and the amount of hydration products. Nonevaporated water comes mainly from the $\mathrm{CH}$ and $\mathrm{C}-\mathrm{S}-\mathrm{H}$ gel of the hydration products. The addition of fly ash to cement pastes results in changes in the nonevaporable water content of the paste. Cement hydration generates $\mathrm{C}-\mathrm{S}-\mathrm{H}$ gel and $\mathrm{CH}$, while the fly ash reaction can also form C-S-H gel by consuming the $\mathrm{CH}$ that is produced by the cement reaction. Therefore, the nonevaporated quantity of water is not an appropriate measure to distinguish the reaction degree of cement and fly ash.

The total nonevaporated quantity of water $\left(\left(W_{n}\right)_{T 1}\right)$ for the fly ash-cement cementitious system can be expressed as

$$
\left(W_{n}\right)_{T 1}=\left(W_{n}\right)_{\mathrm{c}} \cdot m_{\mathrm{c}}+\left(W_{n}\right)_{\mathrm{f}} \cdot m_{\mathrm{f}}
$$

where $\left(W_{n}\right)_{c}$ is the nonevaporable quantity of water for the cement hydration and $\left(W_{n}\right)_{\mathrm{f}}$ is the nonevaporable quantity of water for the fly ash reaction. $m_{c}$ represents the mass fraction of cement, and $m_{\mathrm{f}}$ is the mass fraction of fly ash. Because fly ash improves the effective water-cement ratio and leads to increases in the degree of hydration of the cement, the nonevaporated water of the hydration products generated by cement is

$$
\left(W_{n}\right)_{\mathrm{c}}=\left(W_{n}\right)_{\mathrm{c}-0}+\left(W_{n}\right)_{\mathrm{c}-\mathrm{f}},
$$

where $\left(W_{n}\right)_{\mathrm{c}-0}$ is the nonevaporated quantity of water for pure cement under the same conditions of hydration, and $\left(W_{n}\right)_{\mathrm{c}-\mathrm{f}}$ is the nonevaporable quantity of water as a result of the enhancement in cement hydration due to the presence of fly ash. Equation (3) then becomes

$$
\left(W_{n}\right)_{T 1}=\left[\left(W_{n}\right)_{\mathrm{c}-0}+\left(W_{n}\right)_{\mathrm{c}-\mathrm{f}}\right] \cdot m_{\mathrm{c}}+\left(W_{n}\right)_{\mathrm{f}} \cdot m_{\mathrm{f}} .
$$

The degree of hydration of the fly ash-cement blended materials can be expressed by the following equation:

$$
\alpha_{T 1}=\alpha_{\mathrm{c}} \cdot m+\alpha_{\mathrm{c}-\mathrm{f}} \cdot m_{\mathrm{c}}+\alpha_{\mathrm{f}} \cdot m_{\mathrm{f}} \text {, }
$$

where $\alpha_{T 1}$ is the total degree of hydration of fly ash-cement pastes, $\alpha_{\mathrm{c}}$ is the degree of reaction of pure cement, $\alpha_{\mathrm{f}}$ is the degree of the fly ash reaction, and $\alpha_{c-f}$ is the increase in the degree of hydration of cement due to the presence of fly ash. $\alpha_{c}$ is calculated using the nonevaporated water and the Powers' model, and $\alpha_{\mathrm{f}}$ can be directly determined by selective dissolution in hydrochloric acid. The nonevaporated water of fully hydrated fly ash obtained is 0.168 from the stoichiometry analysis of fly ash reactions [26]. The presence of fly ash leads to an increase in the degree of hydration of the cement $\left(\alpha_{\mathrm{c}-\mathrm{f}}\right)$ that can be calculated by the total nonevaporated water, the nonevaporated water of pure cement, and the nonevaporated water of the fly ash. $\alpha_{\mathrm{c}-\mathrm{f}}$ is obtained from

$$
\begin{aligned}
\alpha_{\mathrm{c}-\mathrm{f}} & =\frac{\left[\left(W_{n}\right)_{T 1}-\left(W_{n}\right)_{\mathrm{c}-0} \cdot m_{\mathrm{c}}-0.168 \cdot \alpha_{\mathrm{f}} \cdot m_{\mathrm{f}}\right]}{\left(0.23 \cdot m_{\mathrm{c}}\right)} \\
\alpha_{\mathrm{f}} & =\frac{\left(w_{n}\right)_{\mathrm{f}}}{0.168} .
\end{aligned}
$$

3.2. Calculation Model of Each Phase Volume Fraction for Fly Ash-Cement Mixtures. Papadakis [30] considered that the glass phase of fly ash mainly consists of active silicon dioxide and aluminum oxide phases that participate in the hydration reactions and generate $\mathrm{C}_{3} \mathrm{~S}_{2} \mathrm{H}_{3}, \mathrm{C}_{4} \mathrm{~A} \overline{\mathrm{S}} \mathrm{H}_{12}$, and $\mathrm{C}_{4} \mathrm{AH}_{13}$. It is assumed that $1 \mathrm{~m}^{3}$ of paste includes $\mathrm{C} \mathrm{kg}$ cement, W kg water, and FA kg fly ash, respectively. $f_{i, \mathrm{C}}$ and $f_{i, \mathrm{FA}}$ represent the mass fraction of oxide $i\left(i=\mathrm{C}(\mathrm{CaO}), \mathrm{S}\left(\mathrm{SiO}_{2}\right), \mathrm{A}\left(\mathrm{Al}_{2} \mathrm{O}_{3}\right)\right.$, $\left.\mathrm{F}\left(\mathrm{Fe}_{2} \mathrm{O}_{3}\right),\left(\mathrm{SO}_{3}\right)\right)$ of cement and fly ash, respectively. $\gamma_{i}$ is the mass fraction of active oxide $i(i=\mathrm{S}, \mathrm{A})$ of fly ash; $R$ is the unreacted content of cement and fly ash, and $H$ is the content of bound water. The model has been proposed according to the stoichiometric reactions for the fly ash-cement blended pastes [30].

When the gypsum content is higher than the amount required to fully hydrate the cement and the activated alumina of the fly ash, $\mathrm{C} \overline{\mathrm{S}} \mathrm{H}_{2}>0.637\left(\mathrm{C}_{3} \mathrm{~A}\right)+1.689(\mathrm{~A})=$ $\left(1.689 f_{\mathrm{A}, \mathrm{c}}-1.078 f_{\mathrm{F}, \mathrm{c}} \mathrm{C}+1.689 \gamma_{\mathrm{A}} f_{\mathrm{A}, \mathrm{FA}} \mathrm{FA}\right.$ or $f_{\overline{\mathrm{s}, \mathrm{c}}}>$ $\left(0.785 f_{\mathrm{A}, \mathrm{C}}-0.501 f_{\mathrm{F}, \mathrm{C}}\right)+0.785 \gamma_{\mathrm{A}} f_{\mathrm{A}, \mathrm{FA}}(\mathrm{FA} / \mathrm{C})$.

The cement reaction occurs as follows:

$$
\begin{array}{r}
2 \mathrm{C}_{3} \mathrm{~S}+10.6 \mathrm{H} \longrightarrow \mathrm{C}_{3.4} \mathrm{~S}_{2} \mathrm{H}_{8}+2.6 \mathrm{CH} \\
2 \mathrm{C}_{2} \mathrm{~S}+8.6 \mathrm{H} \longrightarrow \mathrm{C}_{3.4} \mathrm{~S}_{2} \mathrm{H}_{8}+0.6 \mathrm{CH} \\
\mathrm{C}_{3} \mathrm{~A}+\mathrm{C} \overline{\mathrm{S}} \mathrm{H}_{2}+10 \mathrm{H} \longrightarrow \mathrm{C}_{4} \mathrm{AS}_{\bar{S}} \mathrm{H}_{12} \\
\mathrm{C}_{4} \mathrm{AF}+2 \mathrm{CH}+10 \mathrm{H} \longrightarrow 2 \mathrm{C}_{3}(\mathrm{~A}, \mathrm{~F}) \mathrm{H}_{6}
\end{array}
$$

The fly ash reaction occurs as follows:

$$
\begin{array}{r}
\mathrm{S}+1.1 \mathrm{H}+2.8 \mathrm{H} \longrightarrow \mathrm{C}_{1.1} \mathrm{SH}_{3.9} \\
\mathrm{~A}+\mathrm{C} \overline{\mathrm{S}} \mathrm{H}_{2}+3 \mathrm{CH}+7 \mathrm{H} \longrightarrow \mathrm{C}_{4} \mathrm{~A} \overline{\mathrm{S}} \mathrm{H}_{12}
\end{array}
$$

The quantities of each phase are

$$
\begin{aligned}
\mathrm{CH} & =\left(0.422\left(\mathrm{C}_{3} \mathrm{~S}\right) \alpha_{\mathrm{C}_{3} \mathrm{~S}}+0.129\left(\mathrm{C}_{2} \mathrm{~S}\right) \alpha_{\mathrm{C}_{2} \mathrm{~S}}\right. \\
& \left.-0.305\left(\mathrm{C}_{4} \mathrm{AF}\right) \alpha_{\mathrm{C}_{4} \mathrm{AF}}\right) \mathrm{C}-\left(1.357 \gamma_{\mathrm{S}} f_{\mathrm{s}, \mathrm{FA}}\right. \\
& \left.+2.176 \gamma_{\mathrm{A}} f_{\mathrm{A}, \mathrm{FA}}\right) \alpha_{\mathrm{FA}}(\mathrm{FA})
\end{aligned}
$$




$$
\begin{aligned}
& \mathrm{CSH}=\mathrm{C}_{3.4} \mathrm{~S}_{2} \mathrm{H}_{8}+\mathrm{C}_{1.1} \mathrm{SH}_{3.9}=\left(0.996\left(\mathrm{C}_{3} \mathrm{~S}\right) \alpha_{\mathrm{C}_{3} \mathrm{~S}}\right. \\
& \left.\quad+1.321\left(\mathrm{C}_{2} \mathrm{~S}\right) \alpha_{\mathrm{C}_{2} \mathrm{~S}}\right) \mathrm{C}+3.189 \gamma_{\mathrm{S}} f_{\mathrm{S}, \mathrm{FA}} \alpha_{\mathrm{FA}}(\mathrm{FA}) \\
& \mathrm{CAS} \mathrm{H}=2.304\left(\mathrm{C}_{3} \mathrm{~A}\right) \alpha_{\mathrm{C}_{3} \mathrm{~A}} \mathrm{C}+6.106 \gamma_{\mathrm{A}} f_{\mathrm{A}, \mathrm{FA}} \alpha_{\mathrm{FA}}(\mathrm{FA}) \\
& \mathrm{C}(\mathrm{AF}) \mathrm{H}=1.675\left(\mathrm{C}_{4} \mathrm{AF}\right) \alpha_{\mathrm{C}_{4} \mathrm{AF}} \mathrm{C} \\
& R=\left(1-\left(\mathrm{C}_{3} \mathrm{~S}\right) \alpha_{\mathrm{C}_{3} \mathrm{~S}}-\left(\mathrm{C}_{2} \mathrm{~S}\right) \alpha_{\mathrm{C}_{2} \mathrm{~S}}\right. \\
& \left.\quad-1.637\left(\mathrm{C}_{3} \mathrm{~A}\right) \alpha_{\mathrm{C}_{3} \mathrm{~A}}-\left(\mathrm{C}_{4} \mathrm{AF}\right) \alpha_{\mathrm{C}_{4} \mathrm{AF}}\right) \mathrm{C}+(1 \\
& \left.\quad-\gamma_{\mathrm{S}} f_{\mathrm{S}, \mathrm{FA}} \alpha_{\mathrm{FA}}-2.689 \gamma_{\mathrm{A}} f_{\mathrm{A}, \mathrm{FA}} \alpha_{\mathrm{FA}}\right)(\mathrm{FA}) \\
& H=\left(0.418\left(\mathrm{C}_{3} \mathrm{~S}\right) \alpha_{\mathrm{C}_{3} \mathrm{~S}}+0.450\left(\mathrm{C}_{2} \mathrm{~S}\right) \alpha_{\mathrm{C}_{2} \mathrm{~S}}\right. \\
& \left.+0.667\left(\mathrm{C}_{3} \mathrm{~A}\right) \alpha_{\mathrm{C}_{3} \mathrm{~A}}+0.371\left(\mathrm{C}_{4} \mathrm{AF}\right) \alpha_{\mathrm{C}_{4} \mathrm{AF}}\right) \mathrm{C} \\
& +\left(0.840 \gamma_{\mathrm{S}} f_{\mathrm{S}, \mathrm{FA}} \alpha_{\mathrm{FA}}+1.237 \gamma_{\mathrm{A}} f_{\mathrm{A}, \mathrm{FA}} \alpha_{\mathrm{FA}}\right)(\mathrm{FA})
\end{aligned}
$$

When result of (17) is positive, the pozzolanic reactions of fly ash occur fully; otherwise, there is not enough $\mathrm{CH}$ to react with the $\mathrm{A}$ and $\mathrm{S}$ of the fly ash. When $\mathrm{CH}=0$, the maximum content of fly ash, $\mathrm{FA}_{\max }$, can be obtained.

$$
\begin{aligned}
& \mathrm{FA}_{\max } \\
& =\frac{\left(0.422\left(\mathrm{C}_{3} \mathrm{~S}\right)+0.129\left(\mathrm{C}_{2} \mathrm{~S}\right)-0.305\left(\mathrm{C}_{4} \mathrm{AF}\right)\right) \mathrm{C}}{\left(1.357 \gamma_{\mathrm{S}} f_{\mathrm{s}, \mathrm{FA}}+2.176 \gamma_{\mathrm{A}} f_{\mathrm{A}, \mathrm{FA}}\right)}
\end{aligned}
$$

The volumes of each phase are

$$
\begin{aligned}
& V_{\mathrm{CH}}=\left(0.188\left(\mathrm{C}_{3} \mathrm{~S}\right) \alpha_{\mathrm{C}_{3} \mathrm{~S}}+0.0576\left(\mathrm{C}_{2} \mathrm{~S}\right) \alpha_{\mathrm{C}_{2} \mathrm{~S}}\right. \\
& \left.-0.136\left(\mathrm{C}_{4} \mathrm{AF}\right) \alpha_{\mathrm{C}_{4} \mathrm{AF}}\right) \mathrm{C} \times 10^{-3}-\left(0.606 \gamma_{\mathrm{S}} f_{\mathrm{s}, \mathrm{FA}}\right. \\
& \left.-0.971 \gamma_{\mathrm{A}} f_{\mathrm{A}, \mathrm{FA}}\right) \alpha_{\mathrm{FA}}(\mathrm{FA}) \times 10^{-3} \\
& V_{\mathrm{CSH}}=\left(0.475\left(\mathrm{C}_{3} \mathrm{~S}\right) \alpha_{\mathrm{C}_{3} \mathrm{~S}}+0.630\left(\mathrm{C}_{2} \mathrm{~S}\right) \alpha_{\mathrm{C}_{2} \mathrm{~S}}\right) \mathrm{C} \\
& \times 10^{-3}+1.702 \gamma_{\mathrm{S}} f_{\mathrm{S}, \mathrm{FA}} \alpha_{\mathrm{FA}}(\mathrm{FA}) \times 10^{-3} \\
& V_{\mathrm{CA} \overline{\mathrm{S}} \mathrm{H}}=1.182\left(\mathrm{C}_{3} \mathrm{~A}\right) \alpha_{\mathrm{C}_{3} \mathrm{~A}} \mathrm{C} \times 10^{-3} \\
& +3.131 \gamma_{\mathrm{A}} f_{\mathrm{A}, \mathrm{FA}} \alpha_{\mathrm{FA}}(\mathrm{FA}) \times 10^{-3} \\
& V_{\mathrm{C}(\mathrm{AF}) \mathrm{H}}=0.627\left(\mathrm{C}_{4} \mathrm{AF}\right) \alpha_{\mathrm{C}_{4} \mathrm{AF}} \mathrm{C} \times 10^{-3} \\
& \phi=\frac{W}{\rho_{\mathrm{w}}}-\Delta \phi_{\mathrm{c}}-\Delta \phi_{\mathrm{p}} \\
& \Delta \phi_{\mathrm{c}}=\left(\mathrm{C}_{3} \mathrm{~S}\right) \bar{V}_{\mathrm{C}_{3} \mathrm{~S}}+\left(\mathrm{C}_{2} \mathrm{~S}\right) \bar{V}_{\mathrm{C}_{2} \mathrm{~S}}+\left(\mathrm{C}_{3} \mathrm{~A}\right) \bar{V}_{\mathrm{C}_{3} \mathrm{~A}} \\
& +\left(\mathrm{C}_{4} \mathrm{AF}\right) \bar{V}_{\mathrm{C}_{4} \mathrm{AF}}=\left(0.347\left(\mathrm{C}_{3} \mathrm{~S}\right) \alpha_{\mathrm{C}_{3} \mathrm{~S}}\right. \\
& +0.384\left(\mathrm{C}_{2} \mathrm{~S}\right) \alpha_{\mathrm{C}_{2} \mathrm{~S}}+0.577\left(\mathrm{C}_{3} \mathrm{~A}\right) \alpha_{\mathrm{C}_{3} \mathrm{~A}} \\
& \left.+0.224\left(\mathrm{C}_{4} \mathrm{AF}\right) \alpha_{\mathrm{C}_{4} \mathrm{AF}}\right) \mathrm{C} \times 10^{-3}
\end{aligned}
$$

$$
\begin{aligned}
\Delta \phi_{\mathrm{p}} & =\gamma_{\mathrm{S}} f_{\mathrm{S}, \mathrm{FA}} \alpha_{\mathrm{FA}}(\mathrm{FA}) \bar{V}_{\mathrm{S}}+\gamma_{\mathrm{A}} f_{\mathrm{A}, \mathrm{FA}} \alpha_{\mathrm{FA}}(\mathrm{FA}) \bar{V}_{\mathrm{A}} \\
& =\left(0.635 \gamma_{\mathrm{S}} f_{\mathrm{S}, \mathrm{FA}}+1.180 \gamma_{\mathrm{A}} f_{\mathrm{A}, \mathrm{FA}}\right) \alpha_{\mathrm{FA}}(\mathrm{FA}) \times 10^{-3} \\
\phi & =W \times 10^{-3}-\left(0.347\left(\mathrm{C}_{3} \mathrm{~S}\right) \alpha_{\mathrm{C}_{3} \mathrm{~S}}+0.384\left(\mathrm{C}_{2} \mathrm{~S}\right) \alpha_{\mathrm{C}_{2} \mathrm{~S}}\right. \\
& \left.+0.577\left(\mathrm{C}_{3} \mathrm{~A}\right) \alpha_{\mathrm{C}_{3} \mathrm{~A}}+0.224\left(\mathrm{C}_{4} \mathrm{AF}\right) \alpha_{\mathrm{C}_{4} \mathrm{AF}}\right) \mathrm{C} \\
& \times 10^{-3}-\left(0.635 \gamma_{\mathrm{S}} f_{\mathrm{S}, \mathrm{FA}}+1.180 \gamma_{\mathrm{A}} f_{\mathrm{A}, \mathrm{FA}}\right) \alpha_{\mathrm{FA}}(\mathrm{FA}) \\
& \times 10^{-3} .
\end{aligned}
$$

(2) When the amount of gypsum in the cement is sufficient to hydrate the cement but is not enough to react with all the fly ash activated alumina:

$\mathrm{C} \overline{\mathrm{S}} \mathrm{H}_{2}<0.637\left(\mathrm{C}_{3} \mathrm{~A}\right)+1.689(\mathrm{~A})=\left(1.689 f_{\mathrm{A}, \mathrm{c}}-\right.$ $\left.1.078 f_{\mathrm{F}, \mathrm{c}}\right) \mathrm{C}+1.689 \gamma_{\mathrm{A}} f_{\mathrm{A}, \mathrm{FA}} \mathrm{FA}$ or $f_{\overline{\mathrm{s}, \mathrm{c}}}<\left(0.785 f_{\mathrm{A}, \mathrm{C}}-\right.$ $\left.0.501 f_{\mathrm{F}, \mathrm{C}}\right)+0.785 \gamma_{\mathrm{A}} f_{\mathrm{A}, \mathrm{FA}}(\mathrm{FA} / \mathrm{C})$

The cement reaction occurs as follows:

$$
\begin{array}{r}
2 \mathrm{C}_{3} \mathrm{~S}+10.6 \mathrm{H} \longrightarrow \mathrm{C}_{3.4} \mathrm{~S}_{2} \mathrm{H}_{8}+2.6 \mathrm{CH} \\
2 \mathrm{C}_{2} \mathrm{~S}+8.6 \mathrm{H} \longrightarrow \mathrm{C}_{3.4} \mathrm{~S}_{2} \mathrm{H}_{8}+0.6 \mathrm{CH} \\
\mathrm{C}_{3} \mathrm{~A}+\mathrm{C} \overline{\mathrm{S}} \mathrm{H}_{2}+10 \mathrm{H} \longrightarrow \mathrm{C}_{4} \mathrm{AS}_{\overline{\mathrm{S}}} \mathrm{H}_{12} \\
\mathrm{C}_{4} \mathrm{AF}+2 \mathrm{CH}+10 \mathrm{H} \longrightarrow 2 \mathrm{C}_{3}(\mathrm{~A}, \mathrm{~F}) \mathrm{H}_{6} .
\end{array}
$$

The fly ash reaction occurs as follows:

$$
\begin{array}{r}
\mathrm{S}+1.1 \mathrm{H}+2.8 \mathrm{H} \longrightarrow \mathrm{C}_{1.1} \mathrm{SH}_{3.9} \\
\mathrm{~A}+\mathrm{C} \overline{\mathrm{S}} \mathrm{H}_{2}+3 \mathrm{CH}+7 \mathrm{H} \longrightarrow \mathrm{C}_{4} \mathrm{AS}_{12} \\
\mathrm{~A}+4 \mathrm{CH}+9 \mathrm{H} \longrightarrow \mathrm{C}_{4} \mathrm{AH}_{3}
\end{array}
$$

The quantities of each phase are

$$
\begin{aligned}
& \mathrm{CH}=\left(0.422\left(\mathrm{C}_{3} \mathrm{~S}\right) \alpha_{\mathrm{C}_{3} \mathrm{~S}}+0.129\left(\mathrm{C}_{2} \mathrm{~S}\right) \alpha_{\mathrm{C}_{2} \mathrm{~S}}\right. \\
& \quad-0.305\left(\mathrm{C}_{4} \mathrm{AF}\right) \alpha_{\mathrm{C}_{4} \mathrm{AF}}-0.274\left(\mathrm{C}_{3} \mathrm{~A}\right) \alpha_{\mathrm{C}_{3} \mathrm{~A}} \\
& \left.+0.925 f_{\overline{\mathrm{S}}, \mathrm{C}}\right) \mathrm{C}-\left(1.357 \gamma_{\mathrm{S}} f_{\mathrm{s}, \mathrm{FA}}+2.907 \gamma_{\mathrm{A}} f_{\mathrm{A}, \mathrm{FA}}\right) \\
& \quad \alpha_{\mathrm{FA}}(\mathrm{FA}) \\
& \mathrm{CSH}=\left(0.996\left(\mathrm{C}_{3} \mathrm{~S}\right) \alpha_{\mathrm{C}_{3} \mathrm{~S}}+1.321\left(\mathrm{C}_{2} \mathrm{~S}\right) \alpha_{\mathrm{C}_{2} \mathrm{~S}}\right) \mathrm{C} \\
& \quad+3.189 \gamma_{\mathrm{S}} f_{\mathrm{S}, \mathrm{FA}} \alpha_{\mathrm{FA}}(\mathrm{FA}) \\
& \mathrm{CA} \overline{\mathrm{S}} \mathrm{H}=7.774 f_{\overline{\mathrm{S}}, \mathrm{C}} \mathrm{C} \\
& \mathrm{C}(\mathrm{AF}) \mathrm{H}=1.675\left(\mathrm{C}_{4} \mathrm{AF}\right) \alpha_{\mathrm{C}_{4} \mathrm{AF}} \mathrm{C} \\
& \mathrm{CAH}=\left(2.074\left(\mathrm{C}_{3} \mathrm{~A}\right) \alpha_{\mathrm{C}_{3} \mathrm{~A}}-6.999 f_{\overline{\mathrm{S}}, \mathrm{C}}\right) \mathrm{C} \\
& \quad+5.497 \gamma_{\mathrm{A}} f_{\mathrm{A}, \mathrm{FA}} \alpha_{\mathrm{FA}}(\mathrm{FA})
\end{aligned}
$$




$$
\begin{aligned}
R & =\left(1-\left(\mathrm{C}_{3} \mathrm{~S}\right) \alpha_{\mathrm{C}_{3} \mathrm{~S}}-\left(\mathrm{C}_{2} \mathrm{~S}\right) \alpha_{\mathrm{C}_{2} \mathrm{~S}}-\left(\mathrm{C}_{3} \mathrm{~A}\right) \alpha_{\mathrm{C}_{3} \mathrm{~A}}\right. \\
& \left.-\left(\mathrm{C}_{4} \mathrm{AF}\right) \alpha_{\mathrm{C}_{4} \mathrm{AF}}-2.15 f_{\overline{\mathrm{S}}, \mathrm{C}}\right) \mathrm{C}+\left(1-\gamma_{\mathrm{S}} f_{\mathrm{S}, \mathrm{FA}} \alpha_{\mathrm{FA}}\right. \\
& \left.-\gamma_{\mathrm{A}} f_{\mathrm{A}, \mathrm{FA}} \alpha_{\mathrm{FA}}\right)(\mathrm{FA}) \\
H & =\left(0.418\left(\mathrm{C}_{3} \mathrm{~S}\right) \alpha_{\mathrm{C}_{3} \mathrm{~S}}+0.450\left(\mathrm{C}_{2} \mathrm{~S}\right) \alpha_{\mathrm{C}_{2} \mathrm{~S}}\right. \\
& +0.800\left(\mathrm{C}_{3} \mathrm{~A}\right) \alpha_{\mathrm{C}_{3} \mathrm{~A}}+0.371\left(\mathrm{C}_{4} \mathrm{AF}\right) \alpha_{\mathrm{C}_{4} \mathrm{AF}} \\
& \left.-0.45 f_{\overline{\mathrm{S}}, \mathrm{C}}\right) \mathrm{C}+\left(0.840 \gamma_{\mathrm{S}} f_{\mathrm{S}, \mathrm{FA}} \alpha_{\mathrm{FA}}\right. \\
& \left.+1.591 \gamma_{\mathrm{A}} f_{\mathrm{A}, \mathrm{FA}} \alpha_{\mathrm{FA}}\right)(\mathrm{FA}) .
\end{aligned}
$$

The volumes of each phase are

$$
\begin{aligned}
& V_{\mathrm{CH}}=\left(0.188\left(\mathrm{C}_{3} \mathrm{~S}\right) \alpha_{\mathrm{C}_{3} \mathrm{~S}}+0.058\left(\mathrm{C}_{2} \mathrm{~S}\right) \alpha_{\mathrm{C}_{2} \mathrm{~S}}\right. \\
& -0.136\left(\mathrm{C}_{4} \mathrm{AF}\right) \alpha_{\mathrm{C}_{4} \mathrm{AF}}-0.122\left(\mathrm{C}_{3} \mathrm{~A}\right) \alpha_{\mathrm{C}_{3} \mathrm{~A}} \\
& \left.+0.413 f_{\overline{\mathrm{S}}, \mathrm{C}}\right) \mathrm{C} \times 10^{-3}-\left(0.606 \gamma_{\mathrm{S}} f_{\mathrm{s}, \mathrm{FA}}\right. \\
& \left.+1.298 \gamma_{\mathrm{A}} f_{\mathrm{A}, \mathrm{FA}}\right) \alpha_{\mathrm{FA}}(\mathrm{FA}) \times 10^{-3} \\
& V_{\mathrm{CSH}}=\left(0.474\left(\mathrm{C}_{3} \mathrm{~S}\right) \alpha_{\mathrm{C}_{3} \mathrm{~S}}+0.629\left(\mathrm{C}_{2} \mathrm{~S}\right) \alpha_{\mathrm{C}_{2} \mathrm{~S}}\right) \mathrm{C} \\
& \times 10^{-3}+1.696 \gamma_{\mathrm{S}} f_{\mathrm{S}, \mathrm{FA}} \alpha_{\mathrm{FA}}(\mathrm{FA}) \times 10^{-3} \\
& V_{\mathrm{CA} \overline{\mathrm{S}} \mathrm{H}}=3.987 f_{\overline{\mathrm{S}}, \mathrm{C}} \mathrm{C} \times 10^{-3} \\
& V_{\mathrm{C}(\mathrm{AF}) \mathrm{H}}=0.627\left(\mathrm{C}_{4} \mathrm{AF}\right) \alpha_{\mathrm{C}_{4} \mathrm{AF}} \mathrm{C} \times 10^{-3} \\
& V_{\mathrm{CAH}}=\left(1.001\left(\mathrm{C}_{3} \mathrm{~A}\right) \alpha_{\mathrm{C}_{3} \mathrm{~A}}-3.398 f_{\overline{\mathrm{S}}, \mathrm{C}}\right) \mathrm{C} \times 10^{-3} \\
& +2.668 \gamma_{\mathrm{A}} f_{\mathrm{A}, \mathrm{FA}} \alpha_{\mathrm{FA}}(\mathrm{FA}) \times 10^{-3} \\
& \phi=\frac{W}{\rho_{\mathrm{w}}}-\Delta \phi_{\mathrm{c}}-\Delta \phi_{\mathrm{p}} \\
& \Delta \phi_{\mathrm{c}}=\left(\mathrm{C}_{3} \mathrm{~S}\right) \bar{V}_{\mathrm{C}_{3} \mathrm{~S}}+\left(\mathrm{C}_{2} \mathrm{~S}\right) \bar{V}_{\mathrm{C}_{2} \mathrm{~S}}+\left(\mathrm{C}_{3} \mathrm{~A}\right) \bar{V}_{\mathrm{C}_{3} \mathrm{~A}} \\
& +\left(\mathrm{C}_{4} \mathrm{AF}\right) \bar{V}_{\mathrm{C}_{4} \mathrm{AF}}=\left(0.347\left(\mathrm{C}_{3} \mathrm{~S}\right) \alpha_{\mathrm{C}_{3} \mathrm{~S}}\right. \\
& +0.384\left(\mathrm{C}_{2} \mathrm{~S}\right) \alpha_{\mathrm{C}_{2} \mathrm{~S}}+0.577\left(\mathrm{C}_{3} \mathrm{~A}\right) \alpha_{\mathrm{C}_{3} \mathrm{~A}} \\
& \left.+0.224\left(\mathrm{C}_{4} \mathrm{AF}\right) \alpha_{\mathrm{C}_{4} \mathrm{AF}}\right) \mathrm{C} \times 10^{-3} \\
& \Delta \phi_{\mathrm{p}}=0.635 \gamma_{\mathrm{S}} f_{\mathrm{S}, \mathrm{FA}} \alpha_{\mathrm{FA}}(\mathrm{FA}) \times 10^{-3}+\left(0.075 f_{\overline{\mathrm{S}}, \mathrm{C}}\right. \\
& \left.-0.022\left(\mathrm{C}_{3} \mathrm{~A}\right) \alpha_{\mathrm{C}_{3} \mathrm{~A}}\right) \mathrm{C} \times 10^{-3} \\
& +1.121 \gamma_{\mathrm{A}} f_{\mathrm{A}, \mathrm{FA}} \alpha_{\mathrm{FA}}(\mathrm{FA}) \times 10^{-3} \\
& \phi=W \times 10^{-3}-\left(0.635 \gamma_{\mathrm{S}} f_{\mathrm{S}, \mathrm{FA}}+1.180 \gamma_{\mathrm{A}} f_{\mathrm{A}, \mathrm{FA}}\right) \\
& \text { - } \alpha_{\mathrm{FA}}(\mathrm{FA}) \times 10^{-3}-\left(0.347\left(\mathrm{C}_{3} \mathrm{~S}\right) \alpha_{\mathrm{C}_{3} \mathrm{~S}}\right. \\
& +0.384\left(\mathrm{C}_{2} \mathrm{~S}\right) \alpha_{\mathrm{C}_{2} \mathrm{~S}}+0.555\left(\mathrm{C}_{3} \mathrm{~A}\right) \alpha_{\mathrm{C}_{3} \mathrm{~A}} \\
& \left.+0.224\left(\mathrm{C}_{4} \mathrm{AF}\right) \alpha_{\mathrm{C}_{4} \mathrm{AF}}+0.075 f_{\overline{\mathrm{S}}, \mathrm{C}}\right) \mathrm{C} \times 10^{-3} \text {. }
\end{aligned}
$$
$7 \mathrm{~d}$ of hydration, the nonevaporable water content increased slowly. After $28 \mathrm{~d}$, the nonevaporable water content remained constant for the cement paste samples with $\mathrm{W} / \mathrm{B}$ ratios of 0.3 the degree of the fly ash reaction increased with increasing curing age for various water binder ratios and amounts of fly ash. In the early stage ( $1 \sim 7 \mathrm{~d})$, the fly ash (3\% to $25 \%$, mass fraction) had a greater level of participation in the pozzolanic reaction. Testing the specific surface area and particle size distribution of the fly ash revealed that the fly ash particles were small and their surface area was up to $665 \mathrm{~m}^{2} / \mathrm{kg}$. The outer surface of a large number of fine particles of hydrated fly ash was exposed to $\mathrm{CH}$ in the early stage, and the pozzolanic reactivity of fly ash occurred rapidly. One investigation [31] examined cement-fly ash paste by SEM and found that the surface of many fine fly ash particles appeared to be etched at the age of $7 \mathrm{~d}$ and that hydration products were formed on the surface of the fly ash particles. The present study has shown that a pozzolanic reaction with some of the fly ash began at this stage. When examined at a later age, the degree of the pozzolanic reactions with fly ash had also increased, but the increases gradually slowed.

The effect of fly ash incorporation on the degree of the fly ash reaction at the same water binder ratio (0.5) is shown in Figure 2. The degree of the fly ash reaction decreased with increasing amounts of fly ash. When the fly ash content increased from $10 \%$ to $30 \%, 40 \%$, and $50 \%$ (mass fraction), the degree of the fly ash reaction at $28 \mathrm{~d}$ decreased from $37.1 \%$ to $29.0 \%, 25.4 \%$, and $20.6 \%$, respectively. As the incorporation of fly ash increased, the proportion of cement decreased, and thus the consumption of $\mathrm{CH}$ increased, and its production decreased in the fly ash-cement mixtures. The amount of $\mathrm{CH}$ in the pore solution decreased, and the degree of the fly ash reaction declined. A 10\% content of fly ash showed the highest reaction degree, with $45.15 \%$ at 180 days, whereas the reaction degree of the $50 \%$ content was only $33.11 \%$, indicating that nearly $67 \%$ of the fly ash did not react. Therefore, the filling effect and the microaggregate effect are the major effects when the fly ash amount increases and the pozzolanic reaction is relatively weak.

The effect of the W/B ratio on the degree of the fly ash reaction at the same fly ash incorporation (50\%) is shown in Figure 3. When the W/B ratio rises from 0.3 to 0.5 , the degree of the fly ash reaction exhibited a linearly increasing trend with increased water binder ratio. The average reaction degree of fly ash increased by approximately $11.0 \%$ with each 0.05 increase in the water binder ratio.

4.1.2. Degree of Cement Reaction. The nonevaporable water content of the pure cement pastes changed with curing age, as shown in Figure 4. Curing age had a greater impact on the nonevaporated water content, especially in the early stages (before $28 \mathrm{~d}$ ). From preparation to the curing time of $7 \mathrm{~d}$, the nonevaporable water content increased rapidly. After 

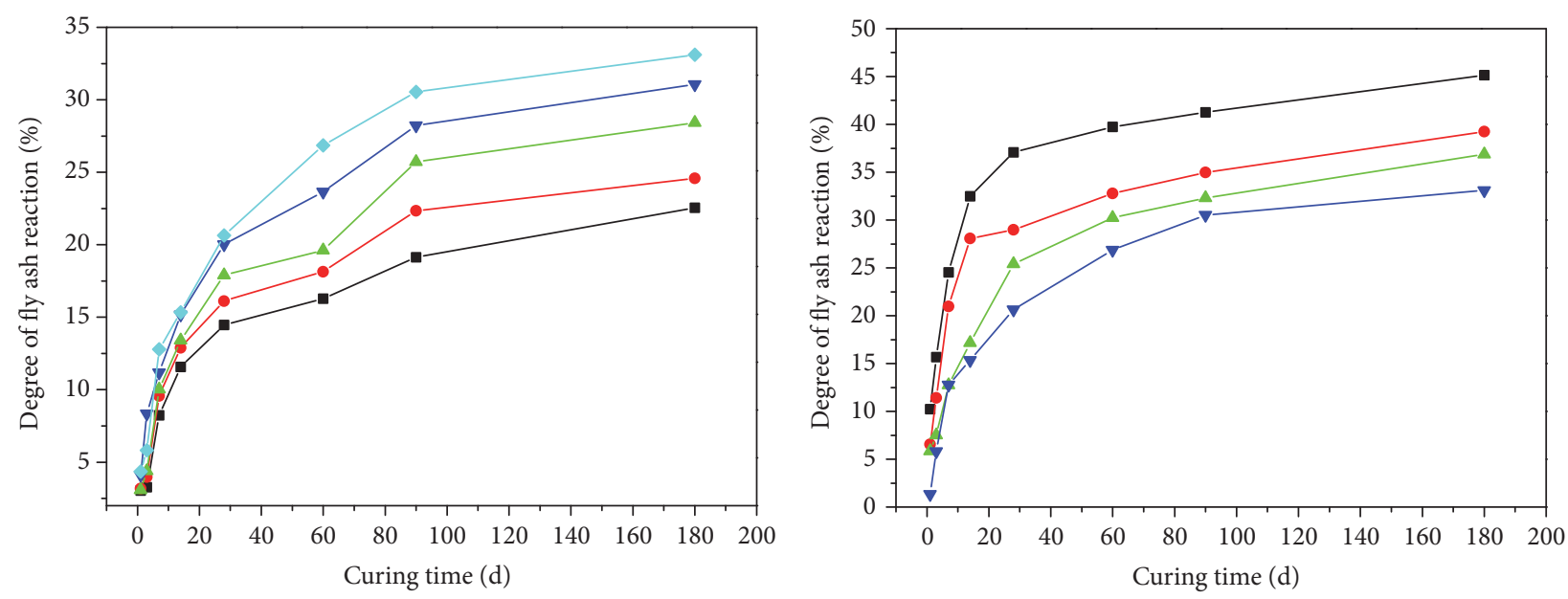

FA50

$$
\begin{aligned}
& \rightarrow \mathrm{W} / \mathrm{B}=0.3 \\
& \rightarrow \mathrm{W} / \mathrm{B}==0.35 \\
& \longrightarrow \mathrm{W} / \mathrm{B}=0.4 \\
& \rightarrow \mathrm{W} / \mathrm{B}=0.45 \\
& \longrightarrow \mathrm{W} / \mathrm{B}=0.5
\end{aligned}
$$

(a) Different $\mathrm{W} / \mathrm{B}$ ratios

$$
\begin{aligned}
& \mathrm{W} / \mathrm{B}=0.5 \\
& \longrightarrow \text { FA10 } \\
& \rightarrow \text { FA30 } \\
& \longrightarrow \text { FA40 } \\
& \rightarrow \text { FA50 }
\end{aligned}
$$

(b) Different amounts of incorporated fly ash

FIGURE 1: Effect of curing time on the degree of the fly ash reaction.

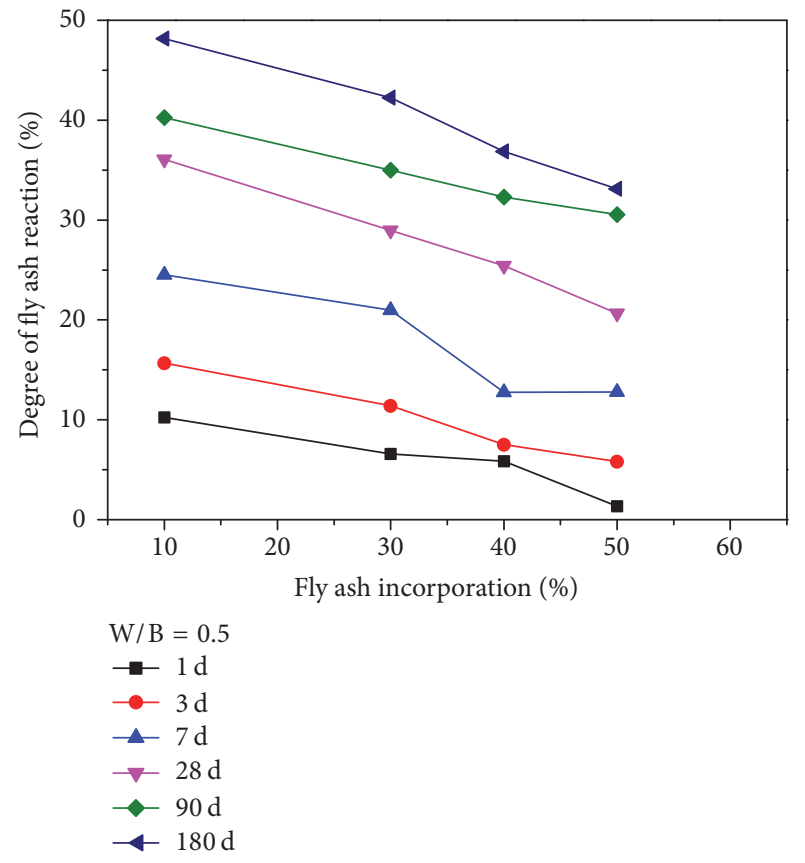

FIGURE 2: Effect of fly ash incorporation on the degree of the fly ash reaction.

and 0.4 , while the nonevaporable water content of the paste with the $\mathrm{W} / \mathrm{B}$ ratio of 0.5 continued to increase.

Based on the experimental results for the nonevaporable water content in the pure cement pastes, the reaction degree of cement $\left(\alpha_{\mathrm{C}}\right)$ can be calculated using (2). In this study, the degree of the cement paste reaction at different $\mathrm{W} / \mathrm{B}$

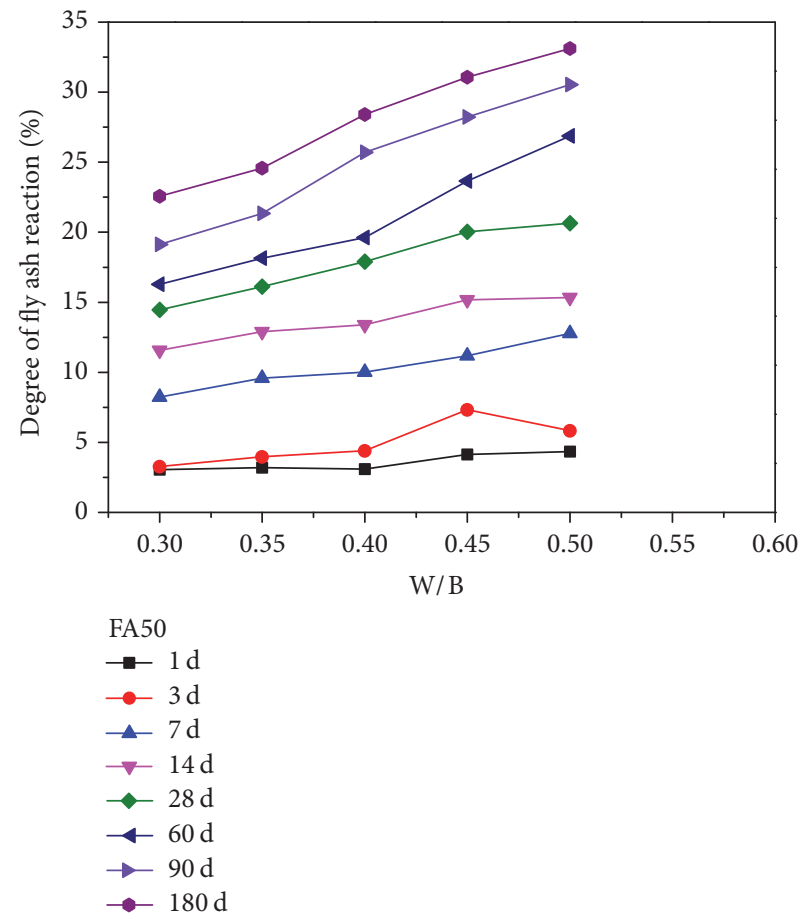

FIgURE 3: Effect of W/B ratio on fly ash reaction degree.

ratios and different curing ages is shown in Figure 5. There is no suitable experimental method to measure the degree of the cement reaction for fly ash-cement pastes at the present time, mainly because the fly ash-cement paste contains not only $\mathrm{C}_{2} \mathrm{~S}_{2} \mathrm{H}, \mathrm{Ca}(\mathrm{OH})_{2}, \mathrm{C}_{3} \mathrm{AH}_{6}$, and AFt from cement 


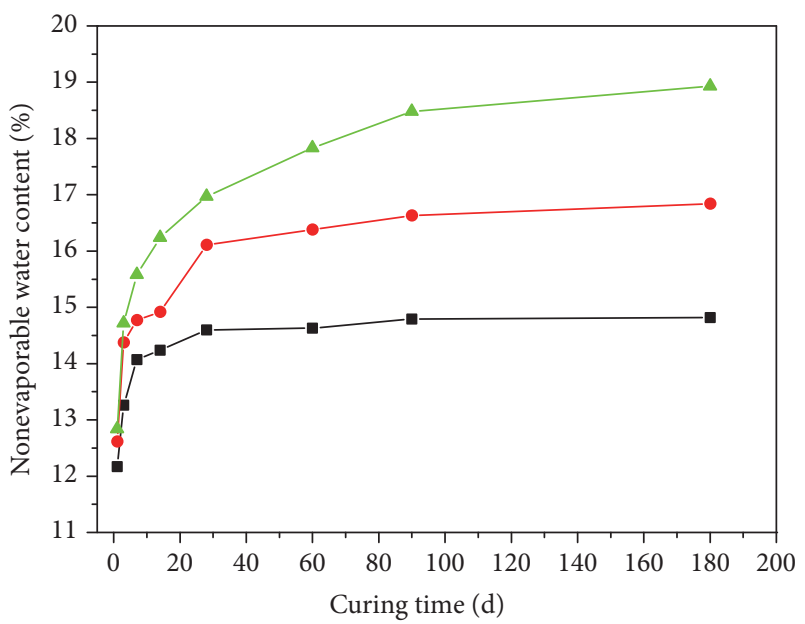

Pure cement pastes

$\rightarrow-W / B=0.3$

$\longrightarrow \mathrm{W} / \mathrm{B}=0.4$

$\rightarrow \mathrm{W} / \mathrm{B}=0.5$

FIgURE 4: Nonevaporable water content of pure cement pastes.

hydration but also includes $\mathrm{C}_{2} \mathrm{~S}_{2} \mathrm{H}, \mathrm{C}_{3} \mathrm{AH}_{6}$, and $\mathrm{AFt}$ from the pozzolanic reactions of fly ash and $\mathrm{Ca}(\mathrm{OH})_{2}$. There are no significant differences in the composition and structure of the $\mathrm{C}_{2} \mathrm{~S}_{2} \mathrm{H}, \mathrm{C}_{3} \mathrm{AH}_{6}$, and $\mathrm{AFt}$ that are formed in the fly ash reactions from those formed in cement hydration. It is difficult to experimentally separate them. Therefore, the traditional methods of determining the hydration degree of pure cement paste have failed by measuring the nonevaporable water and $\mathrm{Ca}(\mathrm{OH})_{2}$ contents of blended pastes. However, quantifying the degree of the cement reaction is the prerequisite for understanding the hydration processes of fly ash-cement pastes. As shown in Figure 2, the degree of cement hydration of pure cement paste depends on the water-cement ratio. An equation describing the relationship between the hydration degree and water-cement ratio is expressed as follows [5]:

$$
\alpha_{\mathrm{c}}=y_{1}(t) e^{-\left(y_{2}(t) /(\mathrm{W} / \mathrm{C})\right)},
$$

where $y_{1}(t)$ and $y_{2}(t)$ are the age-related functions and $\mathrm{W} / \mathrm{C}$ is the water-cement ratio.

To calculate the degree of cement hydration of the fly ashcement system, the water/cement $(\mathrm{W} / \mathrm{C})$ ratio is replaced by the effective water binder ratio in (40). In addition, the fly ash pozzolanic reactions will occur and generate a new product, so the effective water binder ratio is replaced by $\mathrm{W} /\left(\mathrm{C}+\alpha_{\mathrm{f}} \mathrm{FA}\right)$ :

$$
\alpha_{\mathrm{c}}=y_{1}(t) e^{-\left(y_{2}(t) /\left(\mathrm{W} /\left(\mathrm{C}+\alpha_{\mathrm{f}} \mathrm{FA}\right)\right)\right)} \text {. }
$$

The degree of cement hydration of the fly ash-cement system can be calculated under various conditions according to (41), as shown in Figure 6. It was found by comparing the curing times in Figure 6 that the degree of cement hydration was higher than that of pure cement paste under the same conditions when fly ash was mixed into the cement paste. When the fly ash content increased, the hydration degree of cement increased. This is mainly because the incorporation of

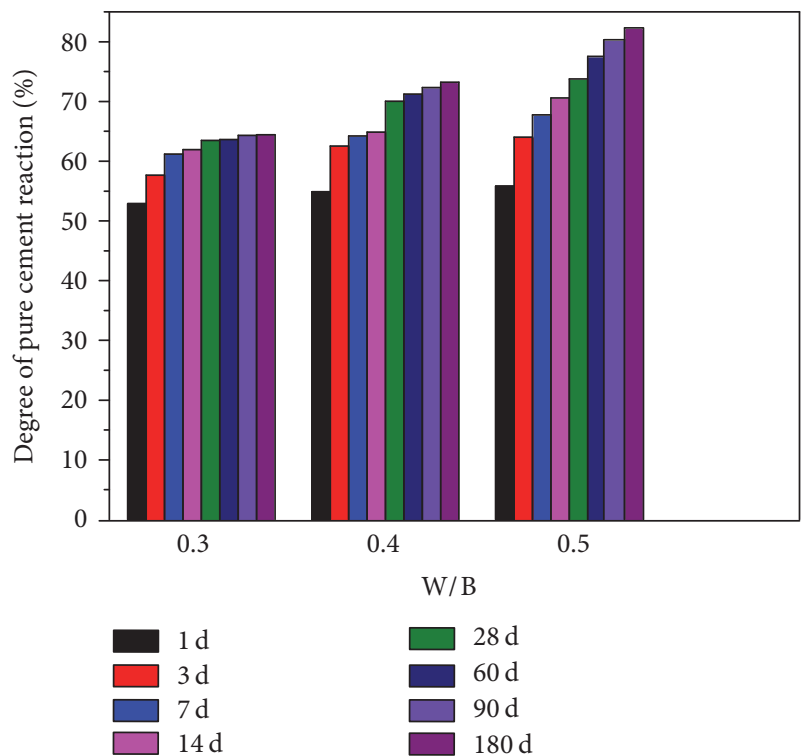

FIGURE 5: Nonevaporable water content of pure cement pastes.

fly ash increased the effective water-cement ratio of cement, improving the hydration environment, and thus increasing the hydration degree. The fly ash contributes to consumption of the hydration product of cement $\left(\mathrm{Ca}(\mathrm{OH})_{2}\right)$, and therefore it is beneficial for the hydration reaction of cement.

4.1.3. Content of Nonevaporable Water. The measured results of nonevaporable water in fly ash-cement blended pastes under various conditions are shown in Figure 7. It can be seen in Figure 7 that the nonevaporated water content of fly ash-cement pastes with $10 \%, 30 \%$, and $40 \%$ fly ash are higher than the pure cement paste in addition to the $50 \%$ content. After $7 \mathrm{~d}$, the differences were not significant. This may be because the nucleation and crystallization of $\mathrm{Ca}(\mathrm{OH})_{2}$ were induced by the fine particles of the fly ash, thus contributing to cement hydration. Within a certain range of incorporation, the promotion effects of fly ash exceeded the negative effects due to the slow development of the activity of fly ash and a small number of hydrates. The nonevaporable water content of fly ash-cement pastes will be higher than that of pure cement pastes. From the nonevaporable water trend, the blended pastes of fly ash incorporation of $10 \sim 30 \%$ showed the highest nonevaporable water. Zhang et al. [32] also found that fly ash can improve the early hydration rate of cement.

Powers [29] proposed that nonevaporable water in pure cement paste is one index for the degree of cement hydration. Nonevaporable water of the hardened pastes comes mainly from the hydration products, $\mathrm{Ca}(\mathrm{OH})_{2}$ and $\mathrm{C}-\mathrm{S}-\mathrm{H}$ gel. In the fly ash-cement blended pastes, both cement hydration and the fly ash reaction produce $\mathrm{C}-\mathrm{S}-\mathrm{H}$, and the fly ash can also consume $\mathrm{Ca}(\mathrm{OH})_{2}$ that is produced by the hydration of the cement. Therefore, it is not appropriate to directly use the nonevaporable water to measure the degree of reaction of the blended pastes. 

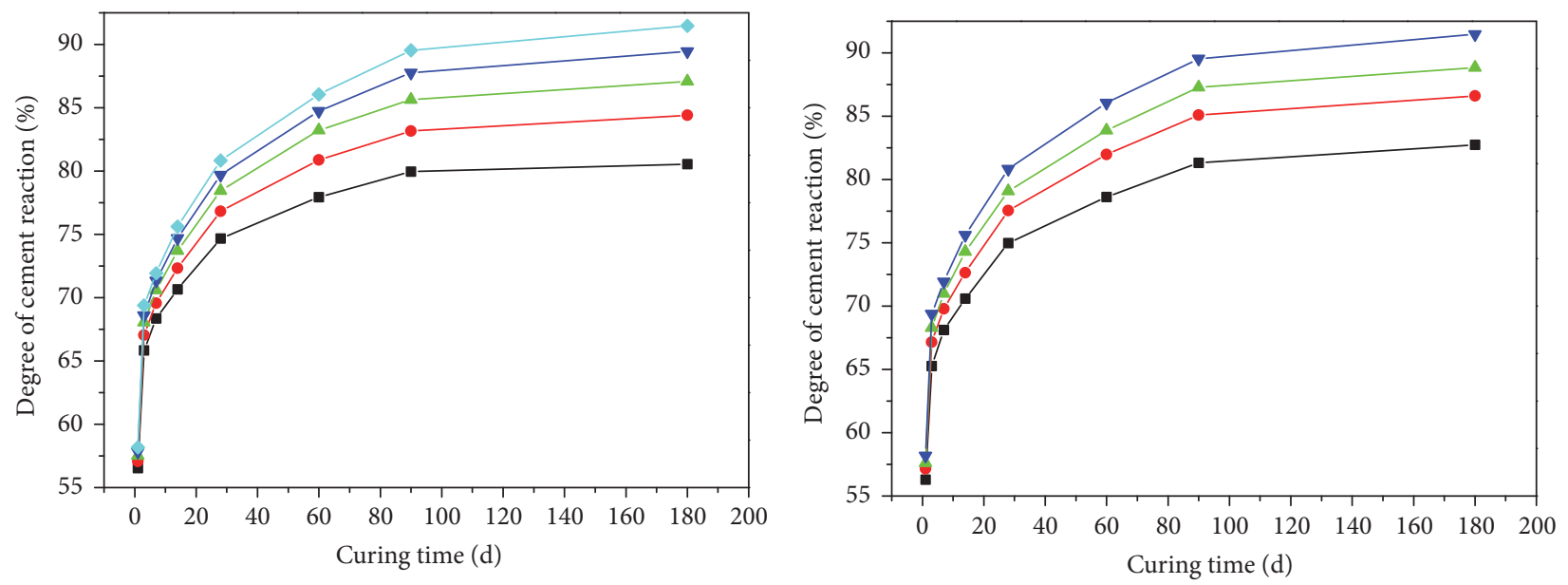

$$
\begin{aligned}
& \text { FA50 } \\
& \rightarrow \mathrm{W} / \mathrm{B}=0.3 \\
& \rightarrow \mathrm{W} / \mathrm{B}=0.35 \\
& \rightarrow \mathrm{W} / \mathrm{B}=0.4 \\
& \rightarrow \mathrm{W} / \mathrm{B}=0.45 \\
& \rightarrow \mathrm{W} / \mathrm{B}=0.5
\end{aligned}
$$

(a) Different $\mathrm{W} / \mathrm{B}$ ratios

$$
\begin{aligned}
& \mathrm{W} / \mathrm{B}=0.5 \\
& - \text { FA10 } \\
& \longrightarrow \text { FA30 } \\
& \longrightarrow \text { - FA40 } \\
& \longrightarrow \text { - FA50 }
\end{aligned}
$$

(b) Different fly ash incorporation

FIGURE 6: The fly ash reaction degree of fly ash-cement system.

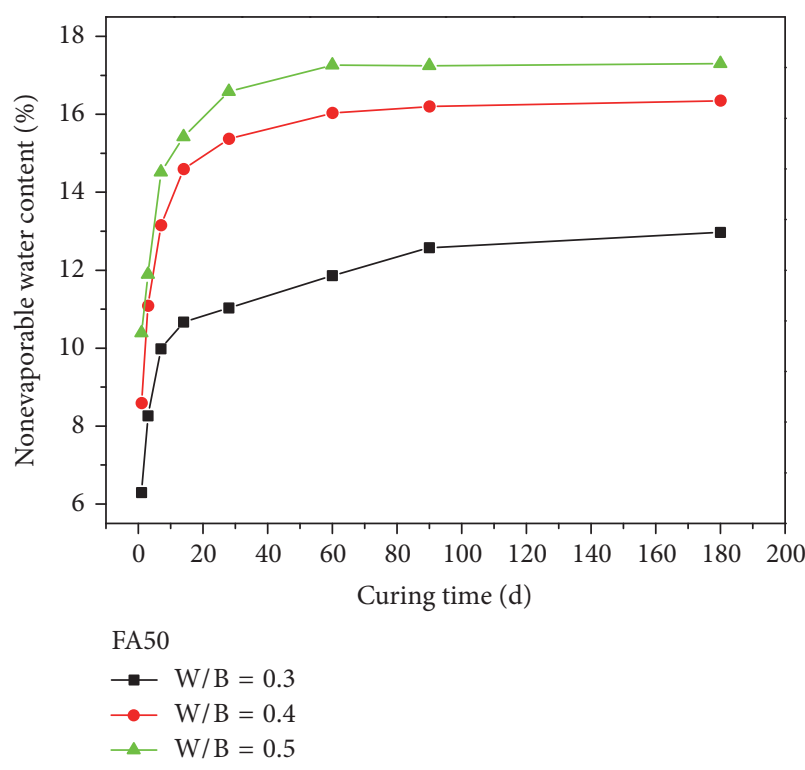

(a) Different $\mathrm{W} / \mathrm{B}$ ratios

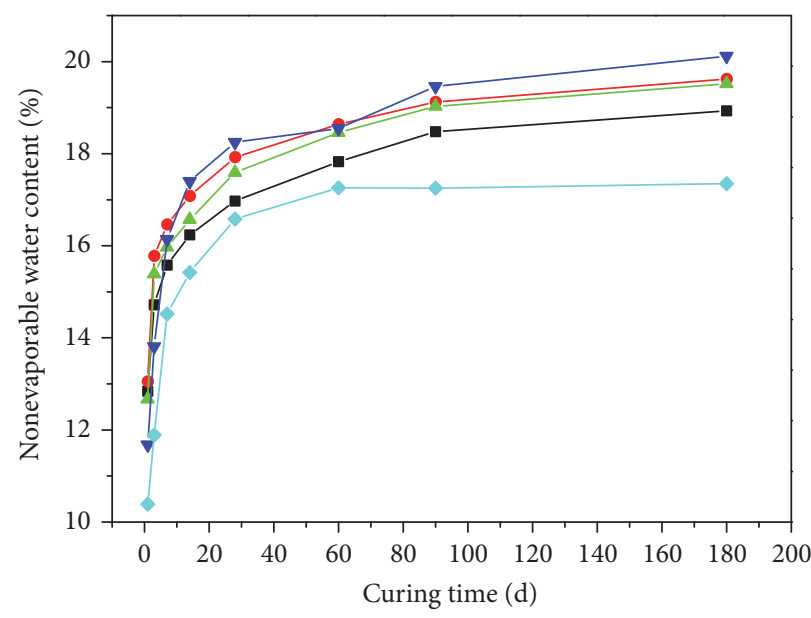

$$
\begin{aligned}
& \mathrm{W} / \mathrm{B}=0.5 \\
& - \text { FA0 } \\
& \longrightarrow \text { FA10 } \\
& \longrightarrow \text { FA30 } \\
& \longrightarrow \text { FA40 } \\
& \longrightarrow \text { FA50 }
\end{aligned}
$$

(b) Different fly ash incorporation rates

FIGURE 7: Nonevaporable water contents of the fly ash-cement blended pastes.

\subsection{Verifying the Model of Fly Ash-Cement Blended Pastes}

4.2.1. The Equations for the Increased Hydration Degree Values of Cement. According to the experimental results of the total amount of nonevaporable water, the degree of the fly ash reaction and the degree of increasing cement hydration were calculated in the fly ash-cement composite systems under various conditions by (7) and (8) as shown in Figures 8 and 9.

Figure 8 shows the variation of the degree of hydration of cement in the blended system with the fly ash addition of $50 \%$ as the $\mathrm{W} / \mathrm{B}$ ratio changed from 0.3 to 0.5 . The reaction degree at every curing age increased linearly with increases of the $\mathrm{W} / \mathrm{B}$ ratio. Figure 9 shows the degree of cement hydration 


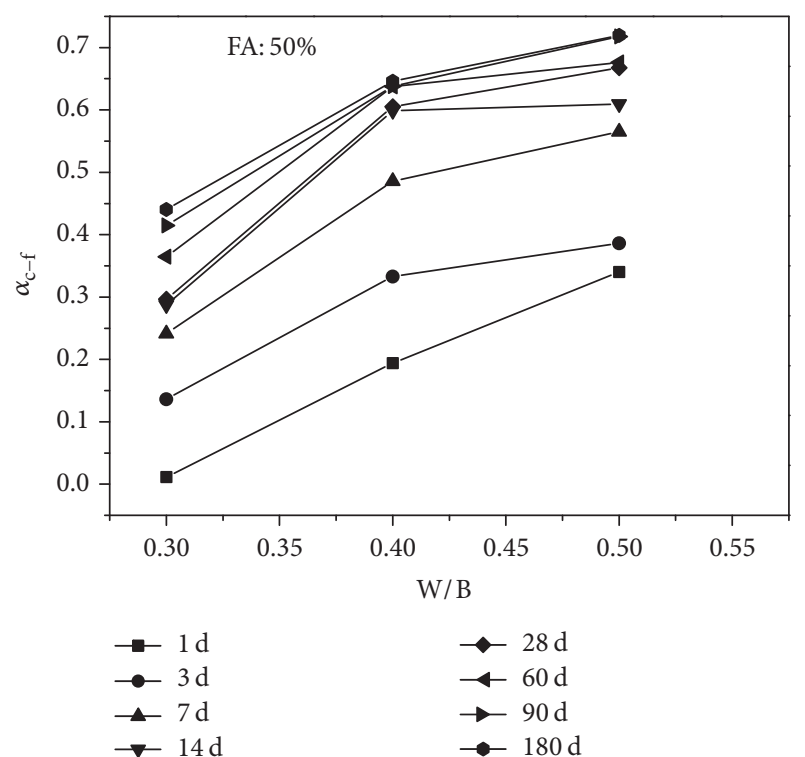

FIgURE 8: Influence of the W/B ratio on the increased degree of cement hydration.

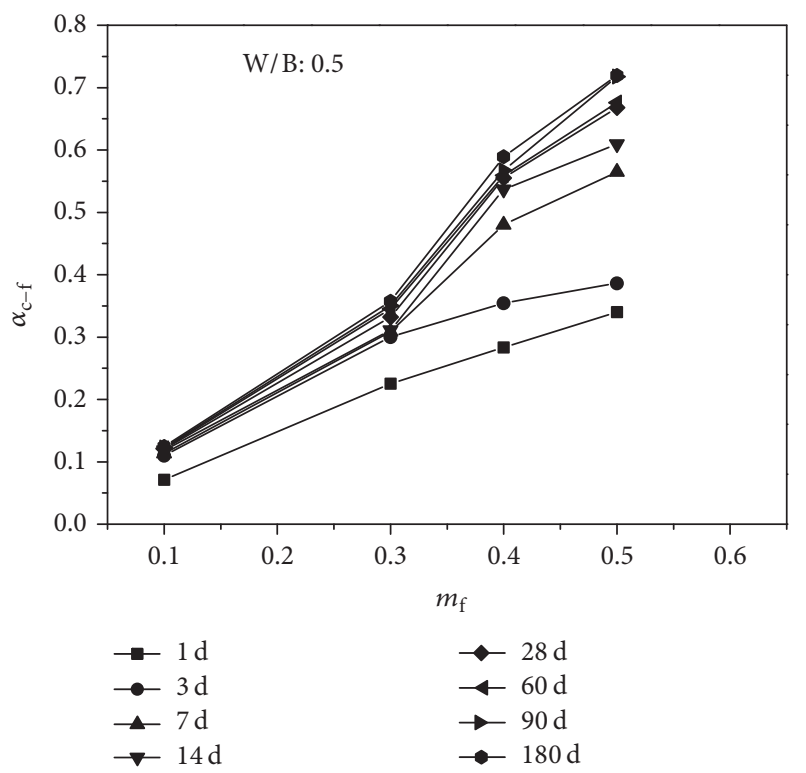

FIGURE 9: Influence of the fly ash content on the increased degree of cement hydration.

with the trend of its fly ash content when the W/B ratio was 0.5 . It can be observed that the added degree of hydration of the cement increased with increases of the fly ash content. When the fly ash content was less than 0.3 , the curing age had little influence on the increased degree of hydration of the cement. When the fly ash content was greater than 0.3 , the degree of hydration of the cement was significantly improved with increasing curing age. For example, as the curing age of the fly ash-cement system increased from $1 \mathrm{~d}$ to $180 \mathrm{~d}$, the value of the degree of hydration of the cement increased from 0.07 to 0.11 when its fly ash content was 0.1 . The increased value of the degree of hydration of cement improved from 0.34 to 0.72 when the fly ash content was 0.5 . Clearly, the W/B ratio, fly ash content, and curing age can each promote the degree of hydration of the cement. On the one hand, this is mainly due to the incorporated fly ash increasing the effective $\mathrm{W} / \mathrm{B}$ ratio of the cement and improving the cement hydration environment. On the other hand, this is due to the postsecondary reactions of fly ash that promptly consume the $\mathrm{Ca}(\mathrm{OH})_{2}$ that is generated by the hydration of the cement, which is advantageous for the hydration reactions of cement.

One objective of this study was to predict the increased degree of hydration of cement and degree of reaction of fly ash in the fly ash-cement blended system with different fly ash contents, W/B ratios, and curing periods. Based on the results of Figures 8 and 9, it is concluded that the increased values of the degree of hydration of cement containing added fly ash and the quantitative relationships among the reaction degree of fly ash, the W/B ratio, and the fly ash content can be expressed as follows, as determined by multiple regression analysis:

$$
\begin{aligned}
\alpha_{\mathrm{c}-\mathrm{f}} & =1-e^{-a(t-b)^{c}} \\
a & =-3.05481+2.86722 \cdot e^{[0.34293 \cdot(\mathrm{W} / \mathrm{B})]} \\
b & =0 \\
c & =-11.67238+11.3153 \cdot e^{\left[0.11795 \cdot\left(m_{\mathrm{f}}\right)\right]} \\
\alpha_{\mathrm{f}} & =1-e^{-d(t-f)^{g}} \\
d & =0.03869+0.00426 \cdot e^{[5.55391 \cdot(\mathrm{W} / \mathrm{B})]} \\
f & =0.9 \\
g & =-0.01487+0.413 \cdot e^{\left[-0.7646 \cdot\left(m_{\mathrm{f}}\right)\right]} .
\end{aligned}
$$

4.2.2. Validation of the Model. Figure 10 shows the comparison of the experimental results and the predicted values for $\alpha_{c-f}$. The fitted $R^{2}$ coefficient of the regression analysis equation $(y=0.99366 x)$ is 0.98849 . Figure 11 shows the $\alpha_{f}$ of the experimental and predicted values, and the fitted $R^{2}$ coefficient of the regression analysis equation $(y=$ $0.99552 x$ ) is 0.99131 . The maximum relative errors of Figures 10 and 11 are $30.96 \%$ and $48.31 \%$, respectively. The model was consistent with the experimental values. Therefore, it is reasonable to propose a model for the degree of the fly ash reaction and a model of accelerated hydration of cement for blended systems.

The $\mathrm{CH}$ content and porosity, as key parameters of the microstructure, were selected to verify the hydration products of fly ash-cement mixtures. The results of the experimental values were obtained from the literature [5]. In this experiment, the $\mathrm{CH}$ content of hydrated cement pastes was determined by thermal gravimetry analysis based on the ignited weight of the sample. The porosity of cement-fly ash pastes was obtained by mercury intrusion porosimetry. The $\mathrm{CH}$ and porosity values are presented in Section 3.2. Figure 12 presents the comparison between the predicted 


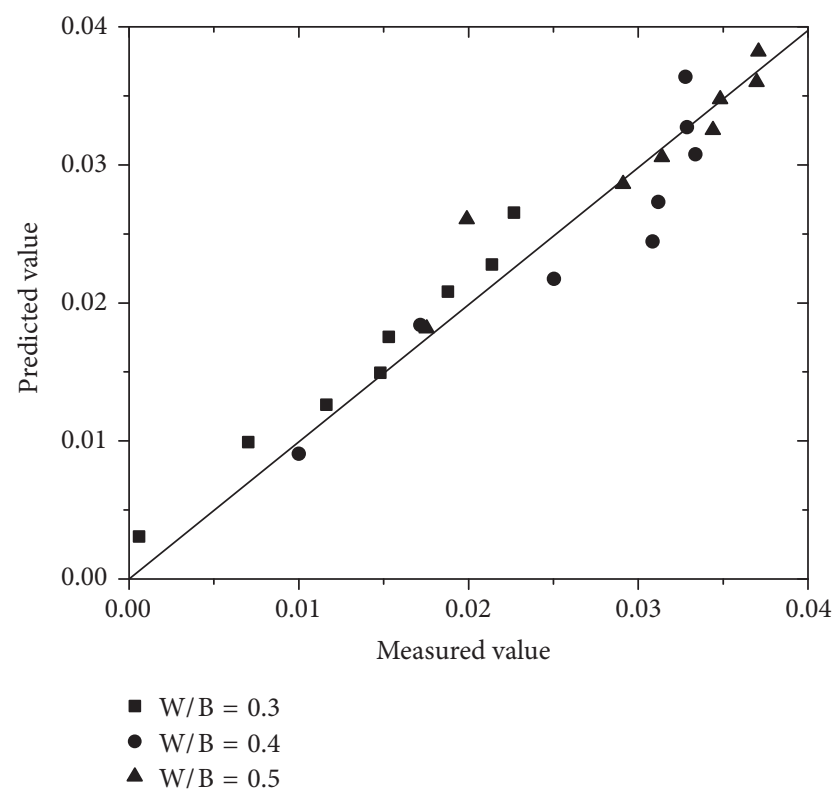

FIGURE 10: The predicted and measured values of the degree of cement hydration of enhanced cement-fly ash pastes.

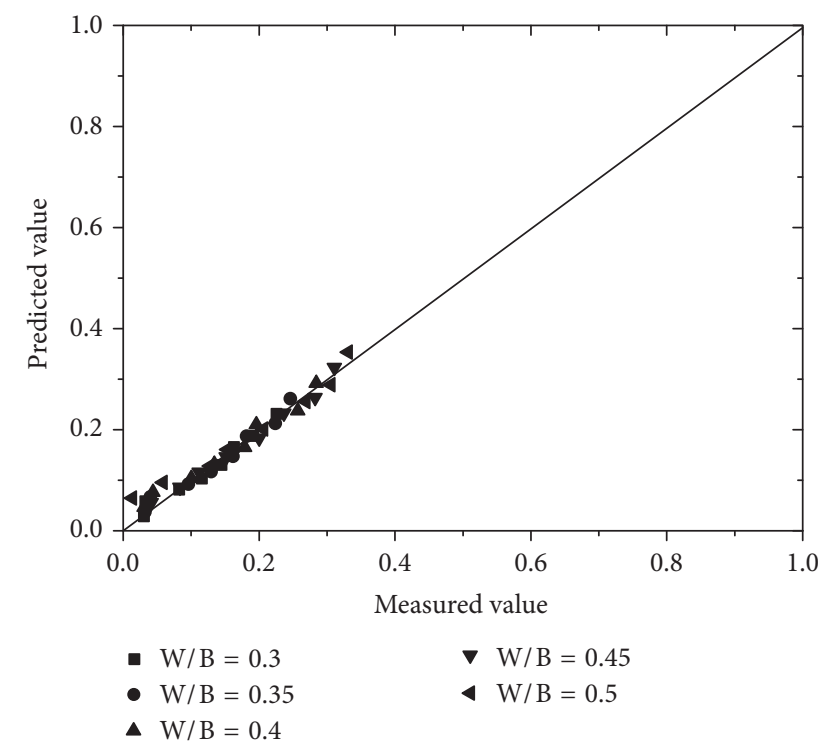

FIGURE 11: The predicted and measured values of the degree of the fly ash reaction of cement-fly ash pastes.

and experimental values of the $\mathrm{CH}$ content. For different $\mathrm{W} / \mathrm{B}$ ratios and fly ash content, the predicted results came closer to the experimental values with increasing curing age. The maximum relative errors were $13.5 \%, 11.3 \%$, and $6.6 \%$ at $7 \mathrm{~d}, 28 \mathrm{~d}$, and $90 \mathrm{~d}$, respectively. Figure 13 shows the comparison of the predicted and experimental values of porosity in fly ash-cement systems. For all samples, the predicted values were higher than the measured values. The maximum relative errors were $11.7 \%, 11.2 \%$, and $13.9 \%$ at $7 \mathrm{~d}$, $28 \mathrm{~d}$, and $180 \mathrm{~d}$, respectively. The main reason is that the porosity of the blended system calculated by the model is

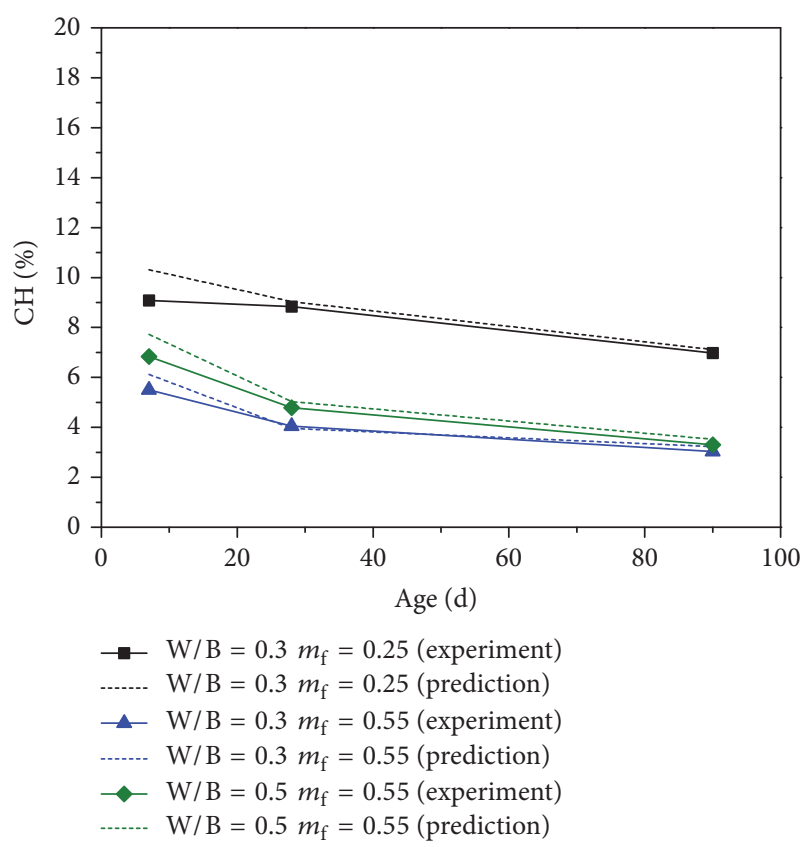

FIGURE 12: The predicted and measured values of $\mathrm{CH}$ content of cement-fly ash pastes.

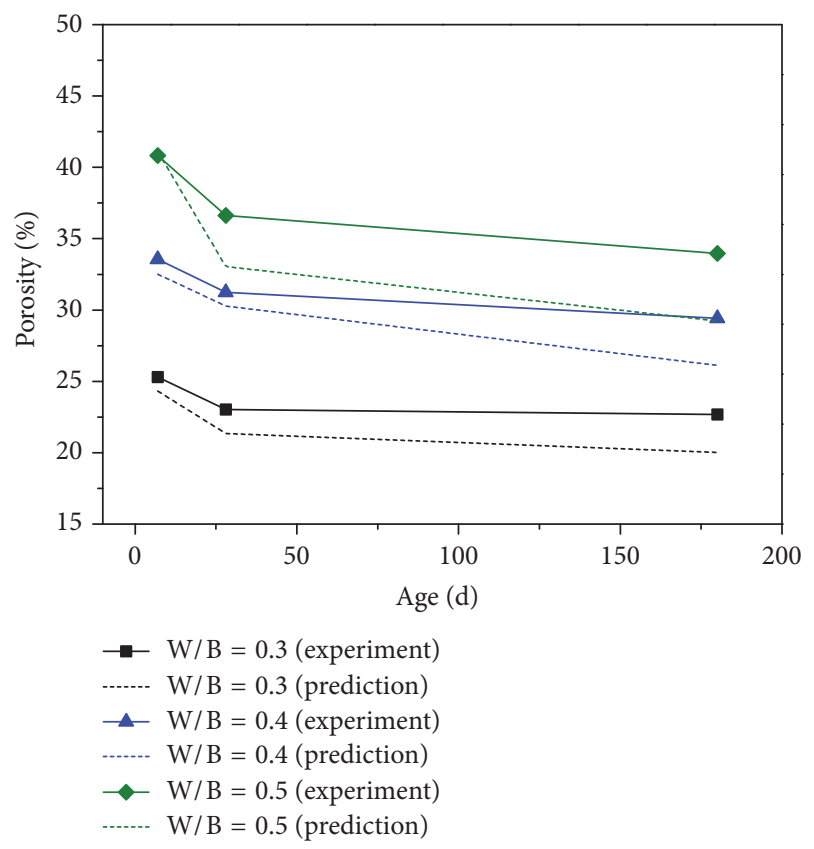

FIGURE 13: The predicted and measured values of porosity of cement-fly ash pastes.

the capillary porosity (the porosity contained in the capillary pores and the porosity of the gel) that was obtained by mercury intrusion. Therefore, with longer curing ages, more $\mathrm{C}-\mathrm{S}-\mathrm{H}$ gel phase was generated by the second hydration of fly ash, and the differences between the predicted and measured values increased. 


\section{Conclusions}

(1) The degree of hydration of cement in the pure cement pastes was determined by measuring the nonevaporable water content. The degree of the fly ash reaction in fly ash-cement blended pastes was determined using a selective dissolution method.

(2) Based on the degree of hydration of cement and the effective $\mathrm{W} / \mathrm{B}$ ratio, the degree of hydration of cement in fly ash-cement blended pastes was acquired.

(3) A hydration model of fly ash-cement blended pastes has been established based on the degree of reaction and the hydration products. This model incorporates the reactions of fly ash and the hydration of cement but is also influenced by their interactions.

\section{Competing Interests}

The authors declare that there are no competing interests regarding the publication of this paper.

\section{Acknowledgments}

The authors gratefully acknowledge the National Natural Science Foundation of China (51408597) and the Fundamental Research Funds for the Central Universities (2014QNA75).

\section{References}

[1] K.-H. Yang, Y.-B. Jung, M.-S. Cho, and S.-H. Tae, "Effect of supplementary cementitious materials on reduction of $\mathrm{CO} 2$ emissions from concrete," Journal of Cleaner Production, vol. 103, pp. 774-783, 2015.

[2] T. Sato and J. J. Beaudoin, "Effect of nano-CaCO3 on hydration of cement containing supplementary cementitious materials," Advances in Cement Research, vol. 23, no. 1, pp. 33-43, 2011.

[3] Z. Liu, Y. Zhang, and Q. Jiang, "Continuous tracking of the relationship between resistivity and pore structure of cement pastes," Construction and Building Materials, vol. 53, pp. 26-31, 2014.

[4] R. Snellings, G. Mertens, and J. Elsen, "Supplementary cementitious materials," Reviews in Mineralogy and Geochemistry, vol. 74, pp. 211-278, 2012.

[5] L. Lam, Y. L. Wong, and C. S. Poon, "Degree of hydration and gel/space ratio of high-volume fly ash/cement systems," Cement and Concrete Research, vol. 30, no. 5, pp. 747-756, 2000.

[6] M. Ahmaruzzaman, "A review on the utilization of fly ash," Progress in Energy and Combustion Science, vol. 36, no. 3, pp. 327-363, 2010.

[7] P. Hou, S. Kawashima, D. Kong, D. J. Corr, J. Qian, and S. P. Shah, "Modification effects of colloidal nanoSiO ${ }_{2}$ on cement hydration and its gel property," Composites Part B: Engineering, vol. 45, no. 1, pp. 440-448, 2013.

[8] J. David Raja Selvam, D. S. Robinson Smart, and I. Dinaharan, "Microstructure and some mechanical properties of fly ash particulate reinforced AA6061 aluminum alloy composites prepared by compocasting," Materials \& Design, vol. 49, pp. 2834, 2013.
[9] R. Feldman, L. R. Prudencio Jr., and G. Chan, "Rapid chloride permeability test on blended cement and other concretes: correlations between charge, initial current and conductivity," Construction and Building Materials, vol. 13, no. 3, pp. 149-154, 1999.

[10] S. W. M. Supit and F. U. A. Shaikh, "Durability properties of high volume fly ash concrete containing nano-silica," Materials and Structures/Materiaux et Constructions, vol. 48, no. 8, pp. 24312445, 2014.

[11] N. Neithalath and J. Jain, "Relating rapid chloride transport parameters of concretes to microstructural features extracted from electrical impedance," Cement and Concrete Research, vol. 40, no. 7, pp. 1041-1051, 2010.

[12] G. Land and D. Stephan, "The influence of nano-silica on the hydration of ordinary Portland cement," Journal of Materials Science, vol. 47, no. 2, pp. 1011-1017, 2012.

[13] J. Justs, M. Wyrzykowski, F. Winnefeld, D. Bajare, and P. Lura, "Influence of superabsorbent polymers on hydration of cement pastes with low water-to-binder ratio," Journal of Thermal Analysis and Calorimetry, vol. 115, no. 1, pp. 425-432, 2014.

[14] Z. Liu, Y. Zhang, Q. Jiang, W. Zhang, and J. Wu, "Solid phases percolation and capillary pores depercolation in hydrating cement pastes," Journal of Materials in Civil Engineering, vol. 26, no. 12, Article ID 04014090, 2014.

[15] B. Uzal and L. Turanl, "Blended cements containing high volume of natural zeolites: properties, hydration and paste microstructure," Cement and Concrete Composites, vol. 34, no. 1, pp. 101-109, 2012.

[16] Y. Kocak and S. Nas, "The effect of using fly ash on the strength and hydration characteristics of blended cements," Construction and Building Materials, vol. 73, pp. 25-32, 2014.

[17] D. D. Nguyen, L. P. Devlin, P. Koshy, and C. C. Sorrell, "Effects of acetic acid on early hydration of Portland cement," Journal of Thermal Analysis and Calorimetry, vol. 123, no. 1, pp. 489-499, 2016.

[18] D. P. Bentz, R. J. Detwiler, E. J. Garboczi, P. Halamickova, and M. Schwartz, "Multi-scale modeling of the diffusivity of mortar and concrete," in Proceedings of the Chloride Penetration into Concrete, L. O. Nilsson and J. P. Ollivier, Eds., pp. 85-94, RILEM, 1997.

[19] M. W. Grutzeck, D. Shi, G. Liu, and S. Kwan, "Computer simulation of interfacial packing in concrete," Journal of Materials Science, vol. 28, no. 13, pp. 3444-3450, 1993.

[20] D. P. Bentz, "Influence of silica fume on diffusivity in cementbased materials. II. Multi-scale modeling of concrete diffusivity," Cement and Concrete Research, vol. 30, no. 7, pp. 1121-1129, 2000.

[21] M. Voltolini, M. C. Dalconi, G. Artioli et al., "Understanding cement hydration at the microscale: new opportunities from 'pencil-beam' synchrotron X-ray diffraction tomography,' Journal of Applied Crystallography, vol. 46, no. 1, pp. 142-152, 2013.

[22] Z. Liu, W. Chen, Y. Zhang, and H. Lv, "A three-dimensional multi-scale method to simulate the ion transport behavior of cement-based materials," Construction \& Building Materials, vol. 120, pp. 494-503, 2016.

[23] B. A. Suprenant and G. Papadopoulos, "Selective dissolution of portland-fly-ash cements," Journal of Materials in Civil Engineering, vol. 3, no. 1, pp. 48-59, 1991.

[24] D. P. Bentz, E. J. Garboczi, and K. A. Snyder, "A hard core/soft shell microstructural model for studying percolation and transport in three-dimensional composite media," NISTIR 6265, U.S. Department of Commerce, 1999. 
[25] X.-Y. Wang, H.-S. Lee, and K.-B. Park, "Simulation of lowcalcium fly ash blended cement hydration," ACI Materials Journal, vol. 106, no. 2, pp. 167-175, 2009.

[26] Q. Zeng, K. Li, T. Fen-Chong, and P. Dangla, "Determination of cement hydration and pozzolanic reaction extents for fly-ash cement pastes," Construction and Building Materials, vol. 27, no. 1, pp. 560-569, 2012.

[27] A. M. Neville, Properties of Concrete, Addison Wesley Longman, Essex, UK, 4th edition, 1996

[28] C. C. Yang, "The relationship between charge passed and the chloride concentrations in anode and cathode cells using the accelerated chloride migration test," Materials and Structures, vol. 36, no. 264, pp. 678-684, 2003.

[29] T. C. Powers, "Structure and physical properties of hardened portland cement paste," Journal of the American Ceramic Society, vol. 41, no. 1, pp. 1-6, 1958.

[30] V. G. Papadakis, "Effect of fly ash on Portland cement systems: part I. Low-calcium fly ash," Cement and Concrete Research, vol. 29, no. 11, pp. 1727-1736, 1999.

[31] A. Xu and S. L. Sarkar, "Microstructural development in highvolume fly-ash cement system," Journal of Materials in Civil Engineering, vol. 6, no. 1, pp. 117-136, 1994.

[32] Y. M. Zhang, W. Sun, and H. D. Yan, "Hydration of high-volume fly ash cement pastes," Cement and Concrete Composites, vol. 22, no. 6, pp. 445-452, 2000. 

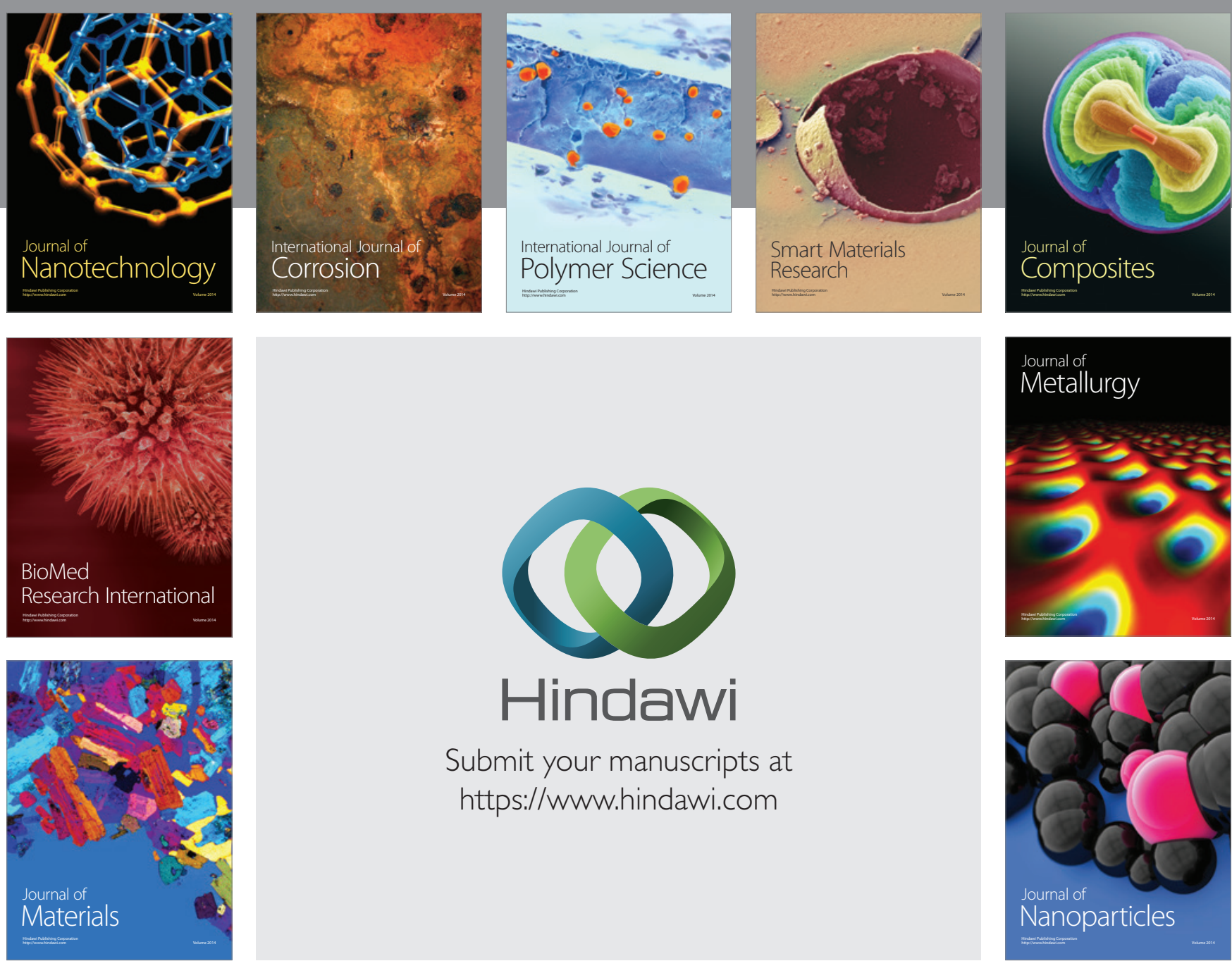

\section{Hindawi}

Submit your manuscripts at

https://www.hindawi.com

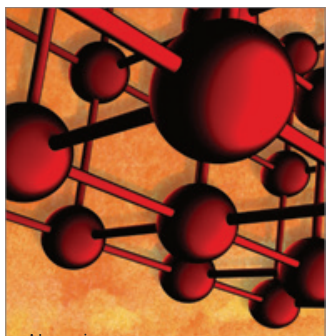

Materials Science and Engineering
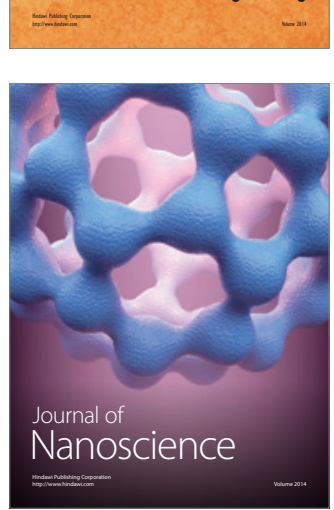
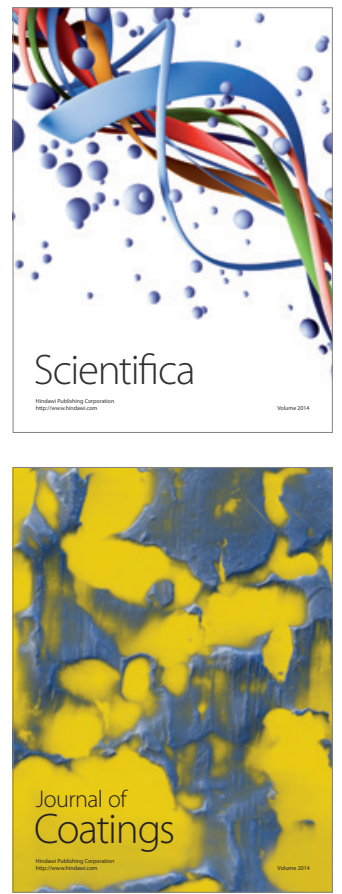
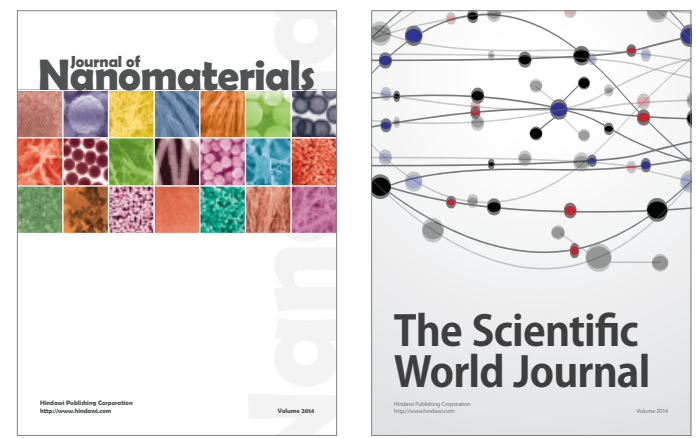

The Scientific World Journal
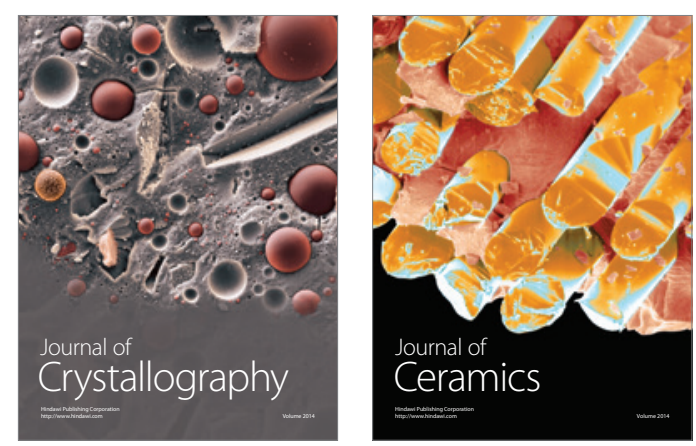
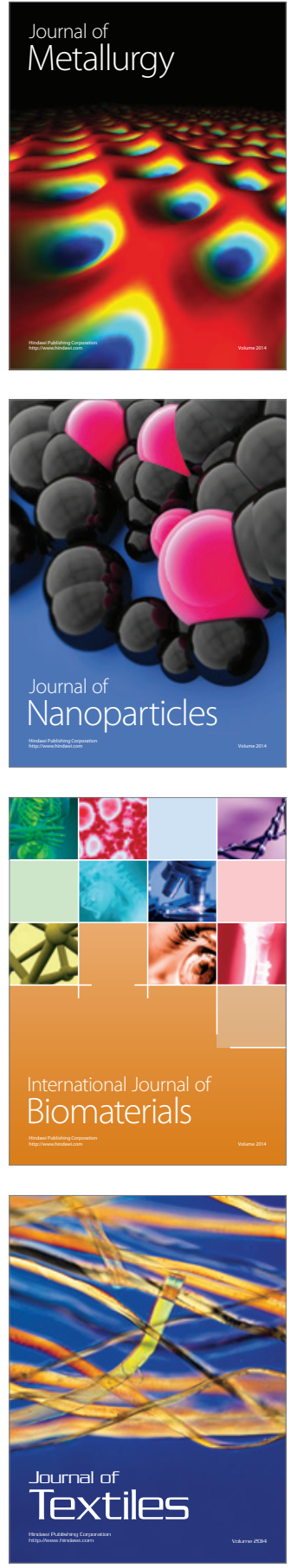